Article

\title{
Biochemical and Hematological Correlates of Elevated Homocysteine in National Surveys and a Longitudinal Study of Urban Adults
}

\author{
May A. Beydoun ${ }^{1, *,+} \mathbb{D}^{D}$, Hind A. Beydoun ${ }^{2}$, Peter H. MacIver ${ }^{1,3}$, Sharmin Hossain ${ }^{1}$, \\ Jose A. Canas ${ }^{4}\left(\mathbb{D}\right.$, Michele K. Evans ${ }^{1, \ddagger}$ and Alan B. Zonderman ${ }^{1, \ddagger}$ \\ 1 Laboratory of Epidemiology and Population Sciences, NIA/NIH/IRP, Baltimore, MD 21224, USA \\ Department of Research Programs, Fort Belvoir Community Hospital, Fort Belvoir, VA 22060, USA \\ Department of Psychology, University of Maryland Baltimore County, Catonsville, MD 21228, USA \\ 4 Department of Pediatrics, Johns Hopkins Medical Institutions, Saint Petersburg, FL 33701, USA \\ * Correspondence: baydounm@mail.nih.gov; Fax: +1-410-558-8236 \\ + M.A.B. had full access to the data used in this manuscript and completed all the statistical analyses. \\ $\ddagger$ Co-senior authors.
}

Received: 12 March 2020; Accepted: 26 March 2020; Published: 30 March 2020

\begin{abstract}
Elevated blood homocysteine (Hcy) among middle-aged adults can increase age-related disease risk, possibly through other biochemical and hematological markers. We selected markers for hyperhomocysteinemia among middle-aged adults, studied time-dependent Hcy-marker associations and computed highly predictive indices of hyperhomocysteinemia, with cross-sectional and longitudinal validations. We used data from the National Health and Nutrition Examination Survey (NHANES III, phase $\left.2, n_{\max }=4000\right)$, the NHANES 1999-2006 $\left(n_{\max }=10,151\right)$ and pooled NHANES (cross-sectional validation). Longitudinal validation consisted of mixed-effects linear regression models (Hcy predicting markers' annual rates of change), applied to the Healthy Aging in Neighborhoods of Diversity Across the Life Span (HANDLS, $n=227-244$ participants, $\mathrm{k}=2.4$ repeats/participant, Age base: 30-65 years) data. Machine learning detected nine independent markers for Hcy > $14 \mu \mathrm{mol} / \mathrm{L}$ (NHANES III, phase 2): older age; lower folate and B-12 status; higher serum levels of creatinine, uric acid, alkaline phosphatase, and cotinine; mean cell hemoglobin and red cell distribution widths (RDW); results replicated in the 1999-2006 NHANES [AUC $=0.60-0.80$ ]. Indices combining binary markers increased elevated Hcy odds by 6.9-7.5-fold. In HANDLS, first-visit Hcy predicted annual increase in creatinine, RDW and alkaline phosphatase, with third-visit index (2013-2018) directly predicting Hcy (2004-2009). We provide evidence of the internal and external validity of indices composed of several biomarkers that are strongly associated with elevated Hcy.
\end{abstract}

Keywords: homocysteine; hematological indices; biochemical indices; inflammation; predictive models; aging

\section{Introduction}

Homocysteine (Hcy) is a sulfur amino acid involved in the remethylation and transsulfuration metabolic pathways, with the first requiring folate and vitamin B- 12 as coenzymes, while the second depends on a form of vitamin B6, pyridoxal 5-phosphate [1]. Epidemiological evidence indicates that elevated Hcy ( $>14 \mu \mathrm{mol} / \mathrm{L})$ can increase risk for cardiovascular and cerebrovascular disease and may double the risk for Alzheimer's Disease (AD) [1-5]. Although biologically plausible, the causal nature of the AD-Hcy association remains a subject of debate. However, predicting Hcy from more commonly measured biochemical and hematological markers and creating a highly predictive index of elevated Hcy can be used in future cohort studies [6,7]. 
During one-carbon metabolism (OCM) cycles, one key enzymatic reaction involves re-methylation of Hcy, whereby a methyl group is acquired from N-5-methyl-tetrahydrofolate (MTHF) or from betaine to form methionine. While the former reaction requires folate and vitamin B12, the latter does not [8]. Adenosine triphosphate (ATP) is then used to convert methionine to S-adenosylemethionine (SAM), a universal methyl donor utilized by various acceptors including nucleic acids, hormones and neurotransmitters [8]. A methyl donation by-product, S-adenosylhomocysteine (SAH), is further hydrolyzed to regenerate Hcy, starting a new cycle of methyl group transfer [8]. The transsulfuration pathway then catabolizes excess Hcy not required for methyl donation into cysteine, using a vitamin-B-6-dependent enzyme, and cysteine is later oxidized to taurine and inorganic sulfates or excreted in urine [8]. Serum folate and vitamin B-6 and B-12 levels are strong inverse predictors of elevated blood Hcy [9-14], as are genetic polymorphisms associated with the OCM, such as MTHFR C667T, associated with reduced methylene tetrahydrofolate reductase (MTHFR) enzymatic activity [15]. Nevertheless, unexplained variability can be ascribed to kidney disease, explaining a positive association between Hcy and serum creatinine [5,16-19]. It is worth noting that men with higher muscle mass have been shown to have higher levels of both Hcy and creatinine, particularly when compared to women, given that $\sim 70 \%$ of daily SAM-dependent methylation reactions are to produce creatine [20,21]. Hcy has been positively associated with red cell distribution width (RDW) [22]; with increased serum cotinine, a measure of active or passive recent smoking [23]; and with increased liver enzyme levels $[24,25]$. Other unexplored biochemical and hematological markers may also be predictive of elevated Hcy which could be reflecting other risk factors for age-related disease such as cardiovascular and neurodegenerative disorders. Generally, there is a paucity of research in the following areas: (1) discovering the most predictive measures of elevated Hcy out of selected biochemical and hematological markers; (2) creating indices that can be used as surrogates of elevated Hcy in studies which do not measure Hcy per se; (3) enhancing understanding as to why elevated Hcy may increase the risk of certain age-related diseases, including $\mathrm{AD}$, by discovering novel markers that are highly predictive of elevated Hcy.

Thus, no study to date has examined and compared potential biochemical and hematological predictors of Hcy among middle-aged adults in a systematic and exploratory manner, by combining machine learning and receiver operating characteristic (ROC) techniques. This novel approach can be applied in other future studies examining the predictors of other clinical mediators of disease. Thus, our present study aimed at selecting a comprehensive yet parsimonious predictive model of elevated Hcy among middle-aged adults, using biochemical and hematological data from the third and most recent (1999-2006) National Health Nutrition Examination Survey, a model cross-validated in a longitudinal study of urban adults, from which an index reflecting elevated Hcy was also validated.

\section{Materials and Methods}

\subsection{Databases}

\subsubsection{NHANES III, Phase 2 and 1999-2006}

The National Health and Nutrition Examination Survey (NHANES) was conducted following guidelines laid down in the Declaration of Helsinki, and all procedures involving human subjects/patients were approved by the Institutional Review Board of the National Center for Health Statistics, Centers for Disease Control and Prevention (CDC). Written or verbal informed consent was obtained from all participants; verbal consent was witnessed and formally recorded [26].

NHANES consists of cross-sectional surveys providing nationally representative data on the health and nutritional status of the U.S. civilian population. Initiated in the 1970s by the National Center for Health Statistics (NCHS), CDC, earlier waves of NHANES collected data in non-continuous fashion. Since 1999, NHANES became a continuous survey. The sampling design is stratified and multistage-probability-clustered. It includes an in-home interview for demographic and basic health information completed by trained staff and a health examination in a mobile examination center 
(MEC), completed by physicians, medical/health technicians, and dietary and health interviewers [26]. Of interest are NHANES waves with complete blood Hcy data, namely NHANES III, phase 2 (1991-1994) [27] and the 1999-2006 wave [26]. Regulations for mandatory fortification of wheat flour with folic acid, currently in place in 53 countries, were implemented in the United States in 1998, adding $140 \mu \mathrm{g}$ of folic acid per $100 \mathrm{~g}$ of enriched cereal grain product, and have been estimated to provide 100-200 $\mu \mathrm{g}$ of folic acid per day to women of childbearing age, ultimately reducing the incidence of neural tube defects [28]. This also resulted in a reduced prevalence of elevated Hcy over time and specifically between the two waves of NHANES used in this study [28].

NHANES specimen storage was consistent across waves. Upon arrival at the CDC or contract laboratories, the frozen specimens were sorted by vial type, and stored initially at $-20 \mathrm{C}$. The refrigerated samples were stored at $4-8 \mathrm{C}$. Frozen specimens whose analysis might have been delayed were stored at $-70 \mathrm{C}$ or lower [29].

\subsubsection{HANDLS 2004-2018}

HANDLS is an ongoing prospective cohort study initiated in 2004. It focuses primarily on disparities in the cardiovascular and cognitive health of a socioeconomically diverse sample of Whites and African Americans aged 30-65 yo at baseline and living in selected neighborhoods of Baltimore, Maryland. In brief, HANDLS used an area probability sampling strategy of thirteen neighborhoods, with details provided elsewhere [30]. Phase 1 of Visit 1 (2004-2009) consisted of screening followed by recruitment, household interviews, while phase 2 of Visit 1 (also 2004-2009) consisted of in-depth examinations in a mobile Medical Research Vehicle (MRV), including measurements of blood pressure; anthropometrics and a fasting blood draw were also collected at the follow-up visits [Visit 2: 2009-2013; Visit 3: 2013-2018]. Although blood Hcy was measured only in a small subset of Visit 1 participants (i.e., at baseline), all other available hematological and biochemical indices had three repeats at Visits 1, 2 and 3 (2004-2009, 2009-2013 and 2013-2018). All clinical laboratory indices were obtained from Quest Diagnostics (Chantilly, VA). Mean follow-up times between visits ranged between 6 months and 8 years, with an average of $4-5$ years.

Participants provided written informed consent after reviewing a protocol booklet written in layman's terms and watching a video detailing all procedures and future re-contacts. The HANDLS study was approved ethically by the Institutional Review Board of the National Institutes of Health, National Institute of Environmental Health Sciences (NIEHS/NIH).

\subsection{Study Samples}

We selected adults aged 30-65 years from the NHANES III (phase 2) and from the 1999-2006 waves. Similarly, by design, Visit 1 of HANDLS consisted of adults aged 30-65 years (Supplementary Figure S1). In the NHANES III, phase 2, biomarkers with $>20 \%$ missing data compared to the sub-sample with complete Hcy measures were excluded. Out of 15,283 participants from phase 2 of NHANES III, 8585 had complete Hcy data, of whom 4008 were in the age range of interest. Of those, 3709-4000 had complete data on up to 82 biochemical and hematological markers. Similarly, for the NHANES 1999-2006, of an initial 41,474 participants, completeness on Hcy data was found for $n=28,449$, of whom 10,151 were aged 30-65 years and the final analytical sample ranged between 9991 and 10,151, after biomarkers were selected with machine learning methods using NHANES III, phase 2 data. Pooling data from NHANES III, phase 2 and NHANES 1999-2006, 14,739-14,829 provided complete data on Hcy and the selected biochemical and hematological indices, within the age range 30-65 years. Finally, out of 3720 HANDLS participants, only 245 individuals had complete data on Hcy measured during the first MRV visit. All these participants had the target Visit 1 age of 30-65 years, and the final analytic samples for longitudinal analysis ranged between 227 and 244 individuals with multiple repeats (up to 3 , mean repeats/participants, $\mathrm{k}=2.4$ ), depending on adjustment levels. 


\subsection{Serum Homocysteine}

In NHANES III, phase 2, serum Hcy was measured at the Jean Mayer USDA Human Nutrition Research Center on Aging, Tufts University, using the high-performance liquid chromatography method of Araki and Sako [31]. In the recent NHANES, serum Hcy was measured using "Abbott Homocysteine (HCY) assay", a fully automated technique [32,33], as was Hcy in the HANDLS sub-cohort. In all datasets, elevated Hcy was defined as $>14 \mu \mathrm{mol} / \mathrm{L}$, a cut-point of 2.639057 on the $\log _{\mathrm{e}}$-transformed scale used to examine the association between elevated Hcy and AD in most previous studies [1-5].

\subsection{Biochemical and Hematological Indices}

Biochemical indices included nutritional biomarkers (e.g., folate, B-12, vitamin D, vitamin E, carotenoids, retinol, vitamin C, total calcium, iron, sodium, potassium etc.), metabolic parameters (e.g., serum insulin, glucose, cholesterol, triglycerides, creatinine, albumin, thyroid hormones, liver enzymes) and inflammatory markers (e.g., C-reactive proteins, Immunoglobulin G (IgG) against specific viruses and bacteria), and environmental indices of air pollution and smoking (e.g., blood lead and serum cotinine).

As stated earlier, lower serum folate and vitamin B-12 concentrations are among the highly predictive markers of elevated Hcy. In NHANES III, phase 2, serum folate and B-12 were measured using Bio-Rad Laboratories "Quantaphase Folate" radioassay kit $[29,34]$, as was the case for more recent NHANES [35,36]. In HANDLS, these two measures were determined using enzyme immunoassay by Quest Diagnostics, Chantilly, VA [37], at Visits 1 through 3. Hematological indices consisted of markers of blood cell counts and characteristics (Supplemental methods 1-3).

\subsection{Covariates}

In all NHANES predictive models, the following covariates were considered: age, sex, race/ethnicity (1: NH white, 2: NH black, 3: Mexican American, 4: other Hispanic, 5: others), poverty status $(0$ : $>125 \%$ of poverty income ratio; $1: \leq 125 \%$ of poverty income ratio), rural vs. urban area of residence, and region (Northeast, Midwest, South and West). Given that the last two factors are fixed in HANDLS (urban, Northeast: Baltimore city), only age, sex, race (African American vs. Whites) and poverty status were included, using a similar cut-point $\leq 125 \%$ of the federal poverty line.

\subsection{Data Handling and Statistical Analysis}

All analyses were conducted using Stata release 16.0 [38]. We first describe study characteristics [covariates (all datasets, waves); Loge-transformed Hcy; Loge-transformed biochemical and hematological indices (NHANES III, phase 2)], overall and by categorical Hcy $(\leq 14 \mu \mathrm{mol} / \mathrm{L}$ vs. $>14 \mu \mathrm{mol} / \mathrm{L})$. Differences in means and proportions across these categories were tested using a design-based F-test accounting for sampling design complexity. Beyond this descriptive step, a multi-stage approach was implemented to select the key predictors of elevated Hcy in NHANES III, phase 2, validate them against the most recent NHANES, and cross-validate those predictors in a longitudinal study of urban adults (HANDLS). A flow diagram is used to summarize this approach (Figure 1).

To select predictive biomarkers of continuous Hcy ( $\log _{\mathrm{e}}$-transformed, z-scored), a statistical learning method known as least absolute shrinkage and selection operator (LASSO) was used. LASSO is a covariate selection methodology that is superior to both generalized linear models without covariate selection and the usually applied stepwise or backward elimination process [39]. In fact, 
stepwise selection is often trapped into a local optimal solution and backward elimination can be time-consuming [39]. The LASSO, which does not ignore stochastic errors, is defined as follows:

$$
\beta(\text { lasso })=\operatorname{argmin} \beta\left\|y-\sum_{j=1}^{p} x_{j} \beta_{j}\right\|^{2}+\lambda \sum_{j=1}^{p}\left|\beta_{j}\right|
$$

with $\lambda$ being a non-negative regularization parameter [39]. The second term of the equation, termed the "11 penalty", is a key portion of this equation, ensuring the success of the LASSO method of covariate selection [39]. In fact, this method was shown to discover the right sparse representation of the model, given certain conditions [39]. More recently, several related methods have been developed and validated against each other, with an adaptive LASSO giving more consistent findings, particularly when compared with the non-negative garotte [39].

In our predictions, we used this convex optimization technique with an $l_{1}$ constraint, known as adaptive LASSO, as the main method to select the final linear regression model for prediction of $\log _{\mathrm{e}}$-transformed Hcy with $\log _{\mathrm{e}}$-transformed and $\mathrm{z}$-scored biomarkers and socio-demographic factors, with the latter being force entered into all models. The model was trained on a random half sample of the total population (among the target age group: 30-65 years, sorting the sample by individual ID and fixing a random seed) and validated against the other half sample to check robustness of findings, by comparing $\mathrm{R}^{2}$ between samples. Adaptive LASSO robustness is then compared to that of cross-validation (cvLASSO) and minimal Bayesian information criterion (minBIC) LASSO, and non-zero parameters were presented for each method. This parsimonious model, with $\log _{\mathrm{e}}$-transformed Hcy as an outcome, was then run on the entire population accounting for survey design complexity (i.e., svy: reg) as a starting point for further backward elimination. Thus, beyond that point, additional terms were eliminated at a type I error of 0.10 . This final model was applied to the binary outcome of elevated blood Hcy ( $>14 \mu \mathrm{mol} / \mathrm{L})$, using svy: logit, and further backward elimination was carried out to obtain a short list of independent predictors for elevated Hcy. As a sensitivity analysis, additional markers identified with adaptive LASSO logistic regression on the same half-sample as for the adaptive LASSO linear regression were included in the reduced model to test their predictive value.

In the full NHANES III, phase 2, the selected $\log _{\mathrm{e}}$-transformed biomarkers and continuous socio-demographic variables (e.g., age) in the final models were then entered into a series of ROC analyses to determine the most appropriate cut-point, which would have the largest sensitivity and specificity in predicting elevated Hcy $(>14 \mu \mathrm{mol} / \mathrm{L})$. Sensitivity (proportion of true positives, i.e., proportion of cases correctly identified as meeting the conditions of elevated Hcy) and specificity (proportion of true negatives, i.e., proportion of non-cases correctly identified as not meeting elevated Hcy) were calculated to evaluate accuracy of selected biochemical and hematological markers in depicting elevated Hcy, creating a series of ROC curves [40,41]. The ROC curve is a graphical plot of sensitivity vs. (1 - specificity) for a binary classifier system as its discrimination threshold is varied. The area underneath of each ROC curve (AUC), a measure that is independent of classifier cut-points, can range between 0 and 1 and be computed with its $95 \%$ confidence interval (95\% CI). 


\begin{tabular}{|c|c|c|c|c|c|c|c|c|}
\hline $\begin{array}{l}\text { Step } \\
\text { 1:Determine } \\
\text { availability of } \\
\text { Hcy }\end{array}$ & $\begin{array}{l}\text { Step 2: Select } \\
\text { out markers } \\
\text { that have <20\% } \\
\text { missing on Hcy }\end{array}$ & $\begin{array}{l}\text { Step 3: } \\
\text { Determine } \\
\text { sample sizes for } \\
\text { complete Hcy } \\
\text { and age range: } \\
\text { 30-65y }\end{array}$ & $\begin{array}{l}\text { Step 4: Descriptive } \\
\text { by elevated Hcy } \\
\text { (>144mollL) in age } \\
\text { range: } 30-65 y\end{array}$ & $\begin{array}{l}\text { Step 5: Split sample into } \\
\text { training and testing set } \\
\text { for LASSO analysis. }\end{array}$ & Step 6: Backward elimination & Step 7: ROC curve analysis & $\begin{array}{l}\text { Step 8: Indices and final } \\
\text { logistic regression model }\end{array}$ & $\begin{array}{l}\text { Step 9: Run mixed effects } \\
\text { linear regression and LOWESS }\end{array}$ \\
\hline $\begin{array}{l}\text { NHANES III, } \\
\text { phase 2, } \\
1991-1994 \text { : } \\
>100 \\
\text { biomarkers } \\
\mathrm{N}=8,585 \text { (all } \\
\text { ages: } 1-90 \mathrm{y} \text { ) }\end{array}$ & $\begin{array}{l}\text { NHANES III, } \\
\text { phase 2, 1991- } \\
\text { 1994: } \\
82 \text { biomarkers of } \\
>100\end{array}$ & $\begin{array}{l}\text { NHANES III, } \\
\text { phase 2, 1991- } \\
\text { 1994: } \\
82 \text { biomarkers } \\
\mathrm{N}=3,709-4,000 \\
(30-65 \mathrm{y})\end{array}$ & $\begin{array}{l}\text { NHANES III, phase 2, } \\
\text { 1991-1994: } \\
\text { Socio-demographics } \\
\text { and } 82 \text { biomarkers } \\
\text { [Note: Hcy and } \\
\text { biomarkers are Loge } \\
\text { transformed] }\end{array}$ & $\begin{array}{l}\text { NHANES III, phase 2, } \\
\text { 1991-1994: } \\
\text { - Run cvLASSO linear } \\
\text { regression on training } \\
\text { half sample. } \\
\text { - Run minBIC LASSO } \\
\text { on training half sample. } \\
\text { - Run adaptive LASSO } \\
\text { on training half sample. } \\
\text { - Compare R2 between } \\
\text { training and test } \\
\text { sample. } \\
\text { - List nonzero terms in } \\
\text { descending order of } \\
\text { effect size for each } \\
\text { LASSO method. } \\
\text { - Run adaptive LASSO } \\
\text { logistic as sensitivity } \\
\text { analysis. }\end{array}$ & $\begin{array}{l}\text { NHANES III, phase 2, 1991- } \\
\text { 1994: } \\
\text { Run a linear model with Loge } \\
\text { transformed Hcy as outcome } \\
\text { and predictors selected from } \\
\text { LASSO linear (adaptive } \\
\text { method) } \\
\text { Use entire eligible sample } \\
\text { and adjust for sampling } \\
\text { design complexity. } \\
\text { Starting from this model, } \\
\text { eliminate terms with p }>0.10, \\
\text { using backward elimination. } \\
\text { Use the reduced model for } \\
\text { Loge(chy) as starting point to } \\
\text { eliminate the model further } \\
\text { with logistic regression on } \\
\text { binary elevated Hcy. } \\
\text { Determine final predictors for } \\
\text { elevated Hcy for the next } \\
\text { step. }\end{array}$ & $\begin{array}{l}\text { NHANES III, phase 2, 1991- } \\
\text { 1994: } \\
\text { Run ROC curve analysis for } \\
\text { Hcy }>14 \text { and compare areas } \\
\text { under the curve between } \\
\text { predictors selected in Step } \\
6 . \\
6 . \quad \text { Determine if AUC>0.55. } \\
\text { - Select those with AUC }>0.55 \\
\text { a sinal predictors. } \\
\text { - Present ROC curve, AUC } \\
\text { and optimal cut-point with } \\
\text { highest sensitivity and } \\
\text { specificity. } \\
\text {. Select only the common } \\
\text { predictors that available in } \\
\text { NHANES } 1999-2006 \text {. }\end{array}$ & $\begin{array}{l}\text { Pooled NHANES III, phase } \\
\text { and 1999-2006: } \\
\text { - Create several indices } \\
\text { combining binary } \\
\text { predictors available in } \\
\text { the pooled sample } \\
\text { andior in the HANDLS } \\
\text { sub-cohort. } \\
\text { - Run ROC curve analysis } \\
\text { for HCy } 14 \text { to determine } \\
\text { optimal cutoff of these } \\
\text { ordinal indices. } \\
\text { - Run multiple logistic } \\
\text { regression model for } \\
\text { Hcy }>14 \text { vs. selected } \\
\text { binary predictors and } \\
\text { another set of models } \\
\text { with binary indices. }\end{array}$ & $\begin{array}{l}\text { HANDLS sub-cohort: } \\
\text { Mixed models with outcome } \\
\text { being Hcy and predictors } \\
\text { being continuous predictors } \\
\text { selected in steps } 7-8 \text {. } \\
\text { - Adjust for other covariates, } \\
\text { including biomarkers, in a } \\
\text { serial manner. } \\
\text { - Determine association } \\
\text { between visit } 1 \text { Hcy and visit } \\
1 \text { markers; and visit } 1 \mathrm{Hcy} \text { vs. } \\
\text { change in these markers } \\
\text { over time. } \\
\text { - Sample sizes } \mathrm{N}=227-244, \\
\mathrm{k}=2.4 \text { repeats/participant. } \\
\text { - Determine association } \\
\text { between visit } 1 \mathrm{Hcy} \text { and } \\
\text { ordinal index at visit } 3 \text { using } \\
\text { LOWESS and Pearson's } \\
\text { correlation, } \mathrm{r}(\mathrm{N}=81)\end{array}$ \\
\hline $\begin{array}{l}\text { NHANES } \\
\text { 1999-2006: } \\
\mathrm{N}=28,449 \text { (all } \\
\text { ages: } 0-85 \mathrm{y} \text { ) }\end{array}$ & $\begin{array}{l}\text { NHANES 1999- } \\
\text { 2006: } \\
>100 \text { biomarkers } \\
\text { with } \\
\text { commonalities } \\
\text { with NHANES III, } \\
\text { phase 2 }\end{array}$ & $\begin{array}{l}\text { NHANES } 1999 . \\
2006: \\
N=10,151 \\
(30-65 y)\end{array}$ & $\begin{array}{l}\text { NHANES, 1999-2006: } \\
\text { Socio-demographics }\end{array}$ & & & $\begin{array}{l}\text { NHANES, 1999-2006: } \\
\text { Run ROC curve analysis } \\
\text { using the predictors } \\
\text { selected in Step } 7 \text { for } \\
\text { NHANES III, phase } 2 \text {. } \\
\text { Compare optimal cut-points } \\
\text { and AUC. }\end{array}$ & $\begin{array}{l}\text { The results of this analysis } \\
\text { can used in other cohorts to } \\
\text { create indices for elevated } \\
\text { Hcy, with high sensitivity } \\
\text { and specificity. }\end{array}$ & $\begin{array}{l}\text { The results of this analysis is a } \\
\text { cross-validation of the results } \\
\text { from the national surveys. }\end{array}$ \\
\hline $\begin{array}{l}\text { HANDLS sub- } \\
\text { cohort: visit } 1 \\
\text { (Hcy) } \\
\mathrm{N}=245(30- \\
65 \mathrm{y})\end{array}$ & $\begin{array}{l}\text { HANDLS sub- } \\
\text { cohort: visits 1- } \\
3:>100 \\
\text { biomarkers with } \\
\text { commmonalities } \\
\text { with NHANES III, } \\
\text { phase 2. }\end{array}$ & $\begin{array}{l}\text { HANDLS sub- } \\
\text { cohort: } \\
\mathrm{N}=245 \text { (30-65y) }\end{array}$ & $\begin{array}{l}\text { HANDLS sub-cohort, } \\
\text { visit 1, 2004-2009: } \\
\text { Socio-demographics }\end{array}$ & & & $\begin{array}{l}\text { Pooled NHANES III, phase } \\
\text { and 1999-2006: } \\
\text { Re-run ROC curve analysis } \\
\text { on the pooled sample of } \\
\text { NHANES III, phase and } \\
\text { 1999-2006, with age: } 30- \\
\text { 65y and complete Hcy. }\end{array}$ & & \\
\hline
\end{tabular}

Figure 1. Flow diagram of predictive modeling using LASSO, ROC curves and multivariable regression modeling. Abbreviations: cvLASSO = cross-validation LASSO; LASSO = least absolute shrinkage and selection operator; LOWESS = Locally weighted regression; HANDLS = Healthy Aging in Neighborhoods of Diversity Across the Life Span; minBIC LASSO = minimum Bayesian information criterion LASSO; NHANES = National Health and Nutrition Examination Surveys; R2 = coefficient of determination. 
A $\log _{\mathrm{e}}$-transformed biomarker positively associated with elevated Hcy would yield an AUC between 0.5 and 1.0. An area of 0.70 , for instance, has the following interpretation: if we randomly select a biomarker from the $\mathrm{Hcy}^{+}$and $\mathrm{Hcy}^{-}$groups, the value of that biomarker will be greater in the $\mathrm{Hcy}^{+}$group than in the Hcy ${ }^{-}$group, 70\% of the time. The ROC curves and their associated AUC are presented, with biomarkers inversely linked to elevated Hcy having their values inverted (i.e., multiplying them by -1). Subsequently, biomarkers retained in the final logistic regression model (selected with machine learning and backward elimination), were further pruned out when ROC AUC was $<0.55$. Thus, only biomarkers with AUC $\geq 0.55$ were retained and their AUC and optimal cut-points presented for NHANES III, phase 2, validated against NHANES 1999-2006 and presented for the pooled NHANES.

An index combining all selected binary biomarkers was computed with a potential to range between 0 and $m^{\prime}$ [number of selected biomarkers with positive criterion: $\geq$ optimal cut-point]. The final index summing categorical biomarkers was computed using revised cut-points from pooled NHANES data. Two ordinal indices were obtained, namely, Index I summing all selected binary biomarkers reflecting elevated Hcy that were available in all selected NHANES waves, and Index II, sub-set of Index I, using only commonly measured biomarkers available in the HANDLS study. Similarly, a ROC analysis was conducted on the pooled NHANES to determine the optimal cut-point for Indices I and II. To determine potential use of those biomarkers as surrogates for elevated Hcy in other large epidemiological studies, a logistic regression model was conducted with each Index and with Index I components entered simultaneously in the pooled NHANES data.

Importantly, the cross-sectional and longitudinal associations of Visit 1 Hcy (2004-2009) on repeated measures of the biochemical and hematological markers (Visits 1 through 3: 2004-2018) that were selected for NHANES (continuous) were tested in a sub-set of HANDLS using multiple mixed-effects linear regression analysis (See Supplemental methods 4). All models were adjusted for Visit 1 age (Model 1), with further adjustment for sex, race and poverty status applied to Model 2, while the full model (Model 3) additionally adjusted for all remaining biomarkers. Finally, Index II, computed using the NHANES cut-point, was computed at Visit 3 and correlated with Hcy at Visit 1 of HANDLS using Pearson's correlation and locally weighted regression (LOWESS) smoother, to assess external validity of the association. Type I error was set at 0.05 with $p<0.10$ considered as a trend or tendency towards an association.

\section{Results}

Table 1 present study sample characteristic distributions, namely Hcy and socio-demographic factors for both NHANES waves and for HANDLS Visit 1, as well as biochemical and hematological correlates for NHANES III, phase 2, overall and stratified by Hcy status. In all samples, age and sex (men vs. women) were consistently associated with elevated Hcy, while poverty was directly associated with elevated Hcy only in NHANES 1999-2006, and both race/ethnicity and poverty status trended towards an association with this binary outcome in the HANDLS sub-cohort. Numerous biochemical and hematological indices were significantly associated with elevated Hcy, including serum cotinine, mean cell hemoglobin (MCH), RDW, blood lead, serum uric acid (SUA), serum creatinine, serum alkaline phosphatase, while others were inversely linked to elevated Hcy, namely serum vitamin E, most serum carotenoids, serum retinyl esters, serum folate and vitamin B-12 $(p<0.05)$. It is worth noting that those associations are crude, not adjusted for socio-demographic factors such as age and sex. Among the known predictive factors, serum folate was shown to have an unadjusted mean of 7.6 in the elevated Hcy group compared to 13.6 in the normal group, suggesting a strong inverse relationship. 
Table 1. Participant characteristics distribution by hyperhomocysteinemia status: NHANES III, phase 2; NHANES 1999-2006 and HANDLS $2004-2018{ }^{1}$.

\begin{tabular}{|c|c|c|c|c|c|c|c|c|c|c|c|c|c|}
\hline & \multicolumn{4}{|c|}{ Overall } & \multicolumn{4}{|c|}{ Hcy $\leq 14 \mu \mathrm{mol} / \mathrm{L}$} & \multicolumn{4}{|c|}{ Hcy $>14 \mu \mathrm{mol} / \mathrm{L}$} & \multirow[t]{2}{*}{$\mathrm{P}_{\mathrm{Hcy}}$} \\
\hline & $n$ & Mean & $\%$ & SE & $n$ & Mean & $\%$ & SE & $n$ & Mean & $\%$ & SE & \\
\hline NHANES III, phase 2: 1991-94 & 4000 & & 100.0 & 0.0 & 3663 & & 91.5 & 0.7 & 337 & & 8.4 & 0.7 & \\
\hline Age (y) & 4000 & 44.3 & & 0.42 & 3663 & 44.0 & & 0.45 & 337 & 47.1 & & 0.76 & 0.001 \\
\hline Sex, $\%$ men & 4000 & & 49.0 & 1.2 & 3663 & & 47.8 & 1.2 & & & 62.8 & 4.9 & 0.008 \\
\hline Race/ethnicity & 4000 & & & & 3663 & & & & 337 & & & & 0.51 \\
\hline NH white & & & 74.8 & 2.1 & & & 74.6 & 2.2 & & & 75.8 & 3.7 & \\
\hline NH black & & & 11.1 & 1.0 & & & 10.9 & 1.1 & & & 13.0 & 1.9 & \\
\hline MA & & & 5.2 & 0.7 & & & 5.4 & 0.7 & & & 3.7 & 0.7 & \\
\hline Other & & & 8.8 & 1.6 & & & 9.0 & 1.6 & & & 7.6 & 2.6 & \\
\hline \multicolumn{14}{|l|}{ Poverty status } \\
\hline$P I R \geq 125 \%$ & 3714 & & 14.0 & 1.9 & 3388 & & 14.0 & 1.9 & 326 & & 14.6 & 2.3 & 0.79 \\
\hline \multicolumn{14}{|l|}{$\mathrm{PIR}<125 \%$} \\
\hline Region & 4000 & & 18.9 & 2.2 & 3663 & & 18.4 & 2.5 & 337 & & 23.8 & 4.8 & 0.30 \\
\hline Northeast & & & 22.6 & 4.4 & & & 22.8 & 4.5 & & & 20.7 & 4.4 & \\
\hline Midwest & & & 36.0 & 7.3 & & & 35.8 & 7.4 & & & 36.9 & 7.6 & \\
\hline South & & & 22.6 & 7.5 & & & 22.9 & 7.6 & & & 18.3 & 6.9 & \\
\hline \multicolumn{14}{|l|}{ West } \\
\hline Urban/Rural & 4000 & & & & 3663 & & & & 337 & & & & 0.59 \\
\hline Urban & & & 50.5 & 7.5 & & & 50.7 & 7.6 & & & 48.2 & 8.2 & \\
\hline Rural & & & 49.6 & 7.5 & & & 49.3 & 7.6 & & & 51.8 & 8.2 & \\
\hline Hcy, $\log _{e}$ & 4000 & +2.17 & & 0.01 & 3663 & +2.10 & & 0.01 & 337 & +2.93 & & 0.04 & $<0.001$ \\
\hline $\begin{array}{l}\text { Selected biochemical and } \\
\text { hematological indices, } \log _{e}\end{array}$ & & $\begin{array}{l}\text { Mean, } \\
\log _{e}\end{array}$ & $\begin{array}{l}\text { Mean, } \\
\exp \end{array}$ & $\begin{array}{c}\mathrm{SE} \\
\log _{\mathrm{e}}\end{array}$ & & $\begin{array}{l}\text { Mean, } \\
\log _{e}\end{array}$ & $\begin{array}{l}\text { Mean, } \\
\exp \end{array}$ & $\begin{array}{c}\mathrm{SE}, \\
\log _{e}\end{array}$ & & $\begin{array}{l}\text { Mean, } \\
\log _{e}\end{array}$ & $\begin{array}{l}\text { Mean, } \\
\exp \end{array}$ & $\begin{array}{c}\mathrm{SE}, \\
\log _{e}\end{array}$ & \\
\hline Serum cotinine, $\mathrm{ng} / \mathrm{mL}$ & 3966 & +0.33 & 1.39 & 0.12 & 3630 & +0.17 & 1.190 & 0.11 & 336 & +2.08 & 8.0 & 0.27 & $<0.001$ \\
\hline Serum vitamin $\mathrm{D}, \mathrm{nmol} / \mathrm{L}$ & 3997 & +4.21 & 67.4 & 0.02 & 3660 & +4.21 & 67.40 & 0.02 & 337 & +4.11 & 60.9 & 0.04 & 0.015 \\
\hline Serum thyroxine, $\mathrm{nmol} / \mathrm{L}$ & 3997 & +4.7 & 109.9 & 0.01 & 3660 & +4.70 & 109.9 & 0.01 & 337 & +4.63 & 102.5 & 0.03 & 0.12 \\
\hline Serum TSH, mU/L & 3925 & +0.42 & 1.52 & 0.03 & 3594 & +0.42 & 1.522 & 0.03 & 331 & +0.41 & 1.506 & 0.06 & 0.94 \\
\hline Serum antimicrosomal $\mathrm{Ab}, \mathrm{U} / \mathrm{mL}$ & 3927 & -0.61 & 0.54 & 0.06 & 3596 & -0.61 & 0.543 & 0.06 & 331 & -0.55 & 0.576 & 0.18 & 0.73 \\
\hline Serum anti-thyroglobulin $\mathrm{Ab}, \mathrm{U} / \mathrm{mL}$ & 3927 & -0.06 & 0.94 & 0.04 & 3596 & -0.06 & 0.942 & 0.04 & 331 & -0.03 & 0.970 & 0.09 & 0.76 \\
\hline White blood cell count & 3998 & +1.94 & 6.96 & 0.01 & 3661 & +1.93 & 6.890 & 0.01 & 337 & +2.01 & 7.463 & 0.03 & 0.015 \\
\hline Lymphocyte percent & 3998 & +3.46 & 31.81 & 0.01 & 3661 & +3.47 & 32.14 & 0.01 & 337 & +3.40 & 29.96 & 0.03 & 0.026 \\
\hline Mononuclear percent & 3920 & +1.67 & 5.310 & 0.04 & 3584 & +1.67 & 5.312 & 0.04 & 336 & +1.69 & 5.419 & 0.05 & 0.77 \\
\hline Granulocyte percent & 3920 & +4.10 & 60.34 & 0.01 & 3584 & +4.10 & 60.34 & 0.01 & 336 & +4.13 & 62.17 & 0.02 & 0.12 \\
\hline Lymphocyte number & 3998 & +0.80 & 2.23 & 0.01 & 3661 & +0.79 & 2.20 & 0.01 & 337 & +0.81 & 2.247 & 0.03 & 0.67 \\
\hline Mononuclear number & 3905 & -0.99 & 0.370 & 0.03 & 3572 & -1.00 & 0.368 & 0.03 & 333 & -0.91 & 0.402 & 0.05 & 0.039 \\
\hline
\end{tabular}


Table 1. Cont.

\begin{tabular}{|c|c|c|c|c|c|c|c|c|c|c|c|c|c|}
\hline & \multicolumn{4}{|c|}{ Overall } & \multicolumn{4}{|c|}{ Hcy $\leq 14 \mu \mathrm{mol} / \mathrm{L}$} & \multicolumn{4}{|c|}{ Hcy $>14 \mu \mathrm{mol} / \mathrm{L}$} & \multirow[t]{2}{*}{$\mathrm{P}_{\text {Hcy }}$} \\
\hline & $n$ & Mean & $\%$ & SE & $n$ & Mean & $\%$ & SE & $n$ & Mean & $\%$ & SE & \\
\hline Granulocyte number & 3920 & +1.43 & 4.18 & 0.02 & 3584 & +1.43 & 4.18 & 0.02 & 336 & +1.53 & 4.618 & 0.03 & 0.017 \\
\hline Red blood cell count, SI & 3997 & +1.55 & 4.71 & 0.00 & 3660 & +1.55 & 4.71 & 0.00 & 337 & +1.55 & 4.711 & 0.00 & 0.74 \\
\hline Hemoglobin, g/L & 3998 & +4.96 & 142.5 & 0.00 & 3661 & +4.95 & 141.2 & 0.00 & 337 & +4.97 & 144.0 & 0.01 & 0.001 \\
\hline Hematocrit, $\mathrm{L} / \mathrm{L}=1$ & 3997 & -0.87 & 0.420 & 0.00 & 3660 & -0.87 & 0.419 & 0.00 & 337 & -0.85 & 0.427 & 0.01 & 0.001 \\
\hline Mean cell volume, fL & 3998 & +4.49 & 89.12 & 0.00 & 3661 & +4.49 & 89.12 & 0.00 & 337 & +4.51 & 90.92 & 0.00 & $<0.001$ \\
\hline Mean cell hemoglobin, pg & 3997 & +3.41 & 30.3 & 0.00 & 3660 & +3.41 & 30.27 & 0.00 & 337 & +3.43 & 30.87 & 0.00 & $<0.001$ \\
\hline Mean cell hemoglobin conc., SI & 3997 & +5.82 & 337.0 & 0.00 & 3660 & +5.82 & 337.0 & 0.00 & 337 & +5.83 & 340.3 & 0.00 & 0.30 \\
\hline Red cell distribution width, \% & 3998 & -2.05 & 0.130 & 0.00 & 3661 & -2.05 & 0.129 & 0.00 & 337 & -2.02 & 0.132 & 0.00 & $<0.001$ \\
\hline Platelet count: SI & 3998 & +5.54 & 254.7 & 0.01 & 3661 & +5.53 & 252.1 & 0.01 & 337 & +5.54 & 254.7 & 0.02 & 0.87 \\
\hline Platelet distribution width, $\%$ & 3973 & +2.81 & 16.61 & 0.00 & 3640 & +2.80 & 16.44 & 0.00 & 333 & +2.80 & 16.44 & 0.00 & 0.94 \\
\hline Mean platelet volume, fL & 3997 & +2.13 & 8.41 & 0.00 & 3660 & +2.13 & 8.41 & 0.00 & 337 & +2.12 & 8.331 & 0.01 & 0.30 \\
\hline Lead, $\mu \mathrm{mol} / \mathrm{L}$ & 3999 & -2.13 & 0.118 & 0.03 & 3662 & -2.15 & 0.116 & 0.04 & 337 & -1.82 & 0.162 & 0.06 & $<0.001$ \\
\hline Erythrocyte protoporphyrin, SI & 3999 & -0.19 & 0.83 & 0.01 & 3662 & -0.18 & 0.84 & 0.01 & 337 & -0.25 & 0.779 & 0.03 & 0.029 \\
\hline Serum iron, $\mu \mathrm{mol} / \mathrm{L}$ & 4000 & +2.71 & 15.02 & 0.01 & 3663 & +2.71 & 15.03 & 0.01 & 337 & +2.73 & 15.33 & 0.04 & 0.67 \\
\hline Serum TIBC, $\mu \mathrm{mol} / \mathrm{L}$ & 3997 & +4.16 & 64.07 & 0.01 & 3660 & +4.15 & 63.43 & 0.01 & 337 & +4.18 & 65.37 & 0.01 & 0.067 \\
\hline Serum ferritin, $\mu \mathrm{mol} / \mathrm{L}$ & 3998 & +4.43 & 83.93 & 0.03 & 3661 & +4.41 & 82.27 & 0.03 & 337 & +4.55 & 94.63 & 0.09 & 0.14 \\
\hline Serum folate, $\mathrm{nmol} / \mathrm{L}$ & 4000 & +2.57 & 13.07 & 0.04 & 3663 & +2.61 & 13.60 & 0.04 & 337 & +2.03 & 7.614 & 0.07 & $<0.001$ \\
\hline $\mathrm{RBC}$ folate, $\mathrm{nmol} / \mathrm{L}$ & 3952 & +6.03 & 415.7 & 0.03 & 3615 & +6.05 & 424.1 & 0.02 & 337 & +5.73 & 307.9 & 0.06 & $<0.001$ \\
\hline Serum vitamin B-12, pmol/L & 3999 & +5.79 & 327.0 & 0.01 & 3662 & +5.81 & 333.6 & 0.01 & 337 & +5.52 & 249.6 & 0.04 & $<0.001$ \\
\hline Serum vitamin C, nmol/L & 3841 & +3.50 & 33.11 & 0.04 & 3510 & +3.54 & 34.47 & 0.04 & 331 & +3.11 & 22.42 & 0.09 & $<0.001$ \\
\hline Serum normalized calcium, mmol/L & 3709 & +0.21 & 1.233 & 0.00 & 3410 & +0.21 & 1.234 & 0.00 & 308 & +0.21 & 1.234 & 0.00 & 0.55 \\
\hline Serum total calcium, nmol/L & 3993 & +0.84 & 2.316 & 0.00 & 3657 & +0.84 & 2.316 & 0.00 & 336 & +0.84 & 2.314 & 0.00 & 0.029 \\
\hline Serum selenium, $\mathrm{nmol} / \mathrm{L}$ & 3977 & +0.47 & 1.599 & 0.01 & 3642 & +0.47 & 1.600 & 0.01 & 335 & +0.51 & 1.665 & 0.02 & 0.004 \\
\hline Serum vitamin $\mathrm{A}, \mu \mathrm{mol} / \mathrm{L}$ & 3993 & +0.66 & 1.934 & 0.01 & 3656 & +0.66 & 1.935 & 0.01 & 337 & +0.67 & 1.954 & 0.02 & 0.57 \\
\hline Serum vitamin $\mathrm{E}, \mu \mathrm{mol} / \mathrm{L}$ & 3993 & +3.26 & 26.05 & 0.01 & 3656 & +3.27 & 26.31 & 0.01 & 337 & +3.16 & 23.571 & 0.02 & $<0.001$ \\
\hline Serum alpha carotene, $\mu \mathrm{mol} / \mathrm{L}$ & 3993 & -2.62 & 0.073 & 0.028 & 3622 & -2.60 & 0.074 & 0.030 & 322 & -2.87 & 0.057 & 0.08 & 0.003 \\
\hline Serum beta carotene, $\mu \mathrm{mol} / \mathrm{L}$ & 3991 & -1.24 & 0.289 & 0.02 & 3656 & -1.22 & 0.295 & 0.02 & 335 & -1.56 & 0.210 & 0.07 & $<0.001$ \\
\hline Serum beta-cryptoxanthin, $\mu \mathrm{mol} / \mathrm{L}$ & 3991 & -1.94 & 0.143 & 0.02 & 3655 & -1.92 & 0.147 & 0.02 & 336 & -2.17 & 0.114 & 0.05 & $<0.001$ \\
\hline Serum lutein/zeaxanthin, $\mu \mathrm{mol} / \mathrm{L}$ & 3992 & -1.07 & 0.343 & 0.01 & 3656 & -1.07 & 0.343 & 0.01 & 336 & -1.15 & 0.317 & 0.03 & 0.003 \\
\hline Serum lycopene, $\mu \mathrm{mol} / \mathrm{L}$ & 3990 & -0.91 & 0.402 & 0.02 & 3655 & -0.90 & 0.407 & 0.02 & 335 & -1.00 & 0.368 & 0.03 & 0.012 \\
\hline Serum retinyl esters, $\mu \mathrm{mol} / \mathrm{L}$ & 3974 & -1.78 & 0.169 & 0.02 & 3641 & -1.75 & 0.174 & 0.02 & 333 & -2.09 & 0.124 & 0.05 & $<0.001$ \\
\hline
\end{tabular}


Table 1. Cont.

\begin{tabular}{|c|c|c|c|c|c|c|c|c|c|c|c|c|c|}
\hline & \multicolumn{4}{|c|}{ Overall } & \multicolumn{4}{|c|}{ Hcy $\leq 14 \mu \mathrm{mol} / \mathrm{L}$} & \multicolumn{4}{|c|}{ Hcy $>14 \mu \mathrm{mol} / \mathrm{L}$} & \multirow[t]{2}{*}{$\mathrm{P}_{\text {Hcy }}$} \\
\hline & $n$ & Mean & $\%$ & SE & $n$ & Mean & $\%$ & $\mathrm{SE}$ & $n$ & Mean & $\%$ & SE & \\
\hline Serum cholesterol, $\mathrm{mmol} / \mathrm{L}$ & 3994 & +1.65 & 5.206 & 0.01 & 3657 & +1.65 & 5.207 & 0.01 & 337 & +1.66 & 5.259 & 0.02 & 0.56 \\
\hline Serum triglycerides, $\mathrm{mmol} / \mathrm{L}$ & 3994 & +0.33 & 1.391 & 0.02 & 3657 & +0.32 & 1.377 & 0.02 & 337 & +0.39 & 1.476 & 0.05 & 0.30 \\
\hline Serum HDL-cholesterol, $\mathrm{mmol} / \mathrm{L}$ & 3972 & +0.20 & 1.221 & 0.01 & 3640 & +0.21 & 1.234 & 0.01 & 332 & +0.17 & 1.185 & 0.02 & 0.12 \\
\hline Serum C-reactive protein, mg/dL & 3983 & -1.20 & 0.301 & 0.02 & 3646 & -1.21 & 0.298 & 0.02 & 337 & -1.19 & 0.304 & 0.04 & 0.65 \\
\hline Serum hepatitis $\mathrm{A} \mathrm{Ab}$ & 4000 & +0.47 & 1.600 & 0.01 & 3663 & +0.47 & 1.600 & 0.01 & 337 & +0.48 & 1.616 & 0.03 & 0.67 \\
\hline Serum hepatitis B core Ab & 4000 & +0.65 & 1.915 & 0.00 & 3663 & +0.65 & 1.916 & 0.00 & 337 & +0.64 & 1.896 & 0.02 & 0.66 \\
\hline Serum hepatitis $C A b$ & 4000 & +0.67 & 1.954 & 0.00 & 3663 & +0.68 & 1.974 & 0.00 & 337 & +0.67 & 1.954 & 0.01 & 0.46 \\
\hline Serum rubella $\mathrm{Ab}, \mathrm{IU}$ & 3885 & +4.31 & 74.44 & 0.04 & 3555 & +4.31 & 74.44 & 0.04 & 330 & +4.26 & 70.81 & 0.13 & 0.74 \\
\hline Serum sodium, $\mathrm{mmol} / \mathrm{L}$ & 3997 & +4.95 & 141.1 & 0.00 & 3641 & +4.94 & 139.77 & 0.00 & 336 & +4.95 & 141.1 & 0.00 & 0.81 \\
\hline Serum potassium, $\mathrm{mmol} / \mathrm{L}$ & 3977 & +1.41 & 4.095 & 0.00 & 3641 & +1.41 & 4.096 & 0.00 & 336 & +1.40 & 4.055 & 0.01 & 0.30 \\
\hline Serum chloride, $\mathrm{mmol} / \mathrm{L}$ & 3977 & +4.64 & 103.5 & 0.00 & 3641 & +4.64 & 103.5 & 0.00 & 336 & +4.64 & 103.5 & 0.00 & 0.35 \\
\hline Serum bicarbonate, $\mathrm{mmol} / \mathrm{L}$ & 4000 & +3.30 & 27.11 & 0.01 & 3663 & +3.30 & 27.11 & 0.01 & 337 & +3.30 & 27.11 & 0.02 & 0.91 \\
\hline Serum total calcium, $\mathrm{mmol} / \mathrm{L}$ & 3977 & +0.83 & 2.293 & 0.00 & 3641 & +0.83 & 2.290 & 0.00 & 336 & +0.84 & 2.320 & 0.00 & 0.021 \\
\hline Serum phosphorus, $\mathrm{mmol} / \mathrm{L}$ & 3977 & +0.09 & 1.094 & 0.01 & 3641 & +0.08 & 1.083 & 0.01 & 336 & +0.10 & 1.105 & 0.01 & 0.10 \\
\hline Serum uric acid, $\mu \mathrm{mol} / \mathrm{L}$ & 3977 & +5.72 & 304.9 & 0.01 & 3641 & +5.71 & 301.87 & 0.01 & 336 & +5.82 & 336.97 & 0.02 & $<0.001$ \\
\hline Serum glucose, $\mathrm{mmol} / \mathrm{L}$ & 3974 & +1.68 & 5.366 & 0.01 & 3639 & +1.68 & 5.366 & 0.01 & 335 & +1.69 & 5.419 & 0.03 & 0.66 \\
\hline Serum blood urea nitrogen, SI & 3977 & +1.55 & 4.711 & 0.01 & 3641 & +1.55 & 4.711 & 0.01 & 336 & +1.54 & 4.664 & 0.02 & 0.49 \\
\hline Serum total bilirubin, $\mu \mathrm{mol} / \mathrm{L}$ & 3977 & +2.25 & 9.487 & 0.02 & 3641 & +2.25 & 9.487 & 0.02 & 336 & +2.30 & 9.974 & 0.04 & 0.18 \\
\hline Serum creatinine, $\mu \mathrm{mol} / \mathrm{L}$ & 3977 & +4.52 & 91.83 & 0.00 & 3641 & +4.52 & 91.83 & 0.00 & 336 & +4.61 & 100.48 & 0.02 & $<0.001$ \\
\hline Serum iron, $\mu \mathrm{mol} / \mathrm{L}$ & 3977 & +2.66 & 14.29 & 0.01 & 3641 & +2.66 & 14.30 & 0.01 & 336 & +2.68 & 14.58 & 0.04 & 0.65 \\
\hline Serum cholesterol, $\mathrm{mmol} / \mathrm{L}$ & 3977 & +1.68 & 5.365 & 0.01 & 3641 & +1.68 & 5.366 & 0.01 & 336 & +1.69 & 5.419 & 0.02 & 0.40 \\
\hline Serum triglycerides, $\mathrm{mmol} / \mathrm{L}$ & 3977 & +0.29 & 1.336 & 0.02 & 3641 & +0.29 & 1.336 & 0.02 & 336 & +0.35 & 1.419 & 0.06 & 0.34 \\
\hline Aspartate aminotransferase, U/L & 3977 & +3.02 & 20.49 & 0.01 & 3641 & +3.02 & 20.49 & 0.01 & 336 & +3.02 & 20.49 & 0.05 & 0.90 \\
\hline Alanine aminotransferase, $\mathrm{U} / \mathrm{L}$ & 3977 & +2.86 & 17.46 & 0.02 & 3641 & +2.87 & 17.63 & 0.02 & 336 & +2.73 & 15.33 & 0.07 & 0.058 \\
\hline Gamma glutamyl transferase, $\mathrm{U} / \mathrm{L}$ & 3976 & +3.17 & 23.80 & 0.02 & 3640 & +3.16 & 23.57 & 0.02 & 336 & +3.31 & 27.38 & 0.07 & 0.056 \\
\hline Serum lactate dehydrogenase, $\mathrm{U} / \mathrm{L}$ & 3976 & +5.10 & 164.0 & 0.01 & 3641 & +5.10 & 164.02 & 0.01 & 335 & +5.10 & 164.02 & 0.01 & 0.86 \\
\hline Serum alkaline phosphatase, U/L & 3977 & +4.37 & 79.04 & 0.01 & 3641 & +4.36 & 78.26 & 0.01 & 336 & +4.52 & 91.83 & 0.02 & $<0.001$ \\
\hline Serum total protein, $\mathrm{g} / \mathrm{L}$ & 3977 & +4.29 & 72.96 & 0.00 & 3641 & +4.29 & 72.97 & 0.00 & 336 & +4.29 & 72.97 & 0.01 & 0.80 \\
\hline Serum albumin, $g / \mathrm{L}$ & 3977 & +3.71 & 40.85 & 0.00 & 3641 & +3.71 & 40.85 & 0.00 & 336 & +3.72 & 41.26 & 0.01 & 0.049 \\
\hline Serum globulin, g/L & 3977 & +3.46 & 31.81 & 0.01 & 3641 & +3.46 & 31.82 & 0.01 & 336 & +3.45 & 31.50 & 0.01 & 0.22 \\
\hline Serum osmolality, $\mathrm{mmol} / \mathrm{kg}$ & 3977 & +5.64 & 281.4 & 0.00 & 3641 & +5.64 & 281.46 & 0.00 & 336 & +5.64 & 281.46 & 0.00 & 0.68 \\
\hline Glycated hemoglobin, \% & 3995 & +1.68 & 5.365 & 0.01 & 3658 & +1.68 & 5.365 & 0.01 & 337 & +1.69 & 5.419 & 0.02 & 0.45 \\
\hline Plasma glucose, $\mathrm{mmol} / \mathrm{L}$ & 3996 & +1.67 & 5.312 & 0.01 & 3659 & +1.67 & 5.312 & 0.01 & 337 & +1.68 & 5.365 & 0.02 & 0.66 \\
\hline Urinary cadmium, nmol/L & 3964 & +1.21 & 3.353 & 0.05 & 3637 & +1.19 & 3.287 & 0.05 & 327 & +1.47 & 4.349 & 0.10 & 0.003 \\
\hline
\end{tabular}


Table 1. Cont.

\begin{tabular}{|c|c|c|c|c|c|c|c|c|c|c|c|c|c|}
\hline & \multicolumn{4}{|c|}{ Overall } & \multicolumn{4}{|c|}{ Hcy $\leq 14 \mu \mathrm{mol} / \mathrm{L}$} & \multicolumn{4}{|c|}{ Hcy $>14 \mu \mathrm{mol} / \mathrm{L}$} & \multirow[t]{2}{*}{$P_{\text {Hcy }}$} \\
\hline & $n$ & Mean & $\%$ & SE & $n$ & Mean & $\%$ & SE & $n$ & Mean & $\%$ & SE & \\
\hline Urinary creatinine, $\mathrm{mmol} / \mathrm{L}$ & 3960 & +2.18 & 8.846 & 0.02 & 3635 & +2.17 & 8.758 & 0.02 & 325 & +2.30 & 9.974 & 0.06 & 0.048 \\
\hline Urinary albumin, $\mu \mathrm{g} / \mathrm{L}$ & 3960 & +1.58 & 4.854 & 0.06 & 3635 & +1.55 & 4.711 & 0.06 & 325 & +1.91 & 6.753 & 0.16 & 0.041 \\
\hline \multirow[t]{2}{*}{ Urinary iodine, $\mu \mathrm{g} / \mathrm{L}$} & 3956 & +2.54 & 12.67 & 0.05 & 3631 & +2.54 & 12.68 & 0.05 & 325 & +2.51 & 12.30 & 0.07 & 0.68 \\
\hline & $n$ & Mean & $\%$ & SE & $n$ & Mean & $\%$ & SE & $n$ & Mean & $\%$ & SE & $\mathrm{P}_{\text {Hcy }}$ \\
\hline NHANES 1999-2006 & 10,151 & & 100.0 & 0.0 & 9704 & & 95.9 & 0.3 & 447 & & 4.1 & 0.3 & \\
\hline Age (y) & 10,151 & 45.8 & & 0.20 & 9704 & 45.6 & & 0.20 & 447 & & 50.4 & 0.6 & $<0.001$ \\
\hline Sex, \% men & 10,151 & & 48.6 & 0.4 & 9704 & & 48.2 & 0.4 & 447 & & 59.9 & 0.4 & 0.005 \\
\hline Race/ethnicity & 7605 & & & & 7260 & & & & & & & & 0.50 \\
\hline NH white & & & 73.0 & 2.1 & & & 73.1 & 2.0 & 345 & & 72.1 & 4.1 & \\
\hline NH black & & & 10.9 & 1.1 & & & 10.7 & 1.0 & & & 16.5 & 2.5 & \\
\hline MA & & & 6.8 & 1.0 & & & 7.0 & 1.0 & & & 3.0 & 0.6 & \\
\hline Other & & & 9.2 & 1.1 & & & 9.3 & 1.1 & & & 8.4 & 2.6 & \\
\hline Poverty status & 9471 & & & & 9060 & & & & & & & & $<0.001$ \\
\hline $\mathrm{PIR} \geq 125 \%$ & & & 81.2 & 0.7 & & & 84.6 & 0.7 & 411 & & 75.1 & 2.4 & \\
\hline $\mathrm{PIR}<125 \%$ & & & 15.8 & 0.7 & & & 15.4 & 0.7 & & & 24.9 & 2.4 & \\
\hline \multirow[t]{2}{*}{ Hcy, $\log _{e}$} & 10,151 & +2.08 & & 0.01 & 9704 & +2.04 & & 0.01 & 447 & +2.94 & & 0.02 & $<0.001$ \\
\hline & $n$ & Mean & $\%$ & SE & $n$ & Mean & $\%$ & SE & $n$ & Mean & $\%$ & SE & $\mathrm{P}_{\text {Hcy }}$ \\
\hline HANDLS 2004-2018 & 245 & & 100.0 & & 220 & & 89.8 & & 25 & & 10.2 & & \\
\hline Age (y) & 245 & 49.2 & & 0.56 & 220 & 48.6 & & 0.59 & 25 & 54.3 & & 1.5 & 0.002 \\
\hline Sex, \% men & 245 & & 51.0 & & 220 & & 48.6 & & 25 & & 72.0 & & 0.032 \\
\hline Race/ethnicity & 245 & & & & 220 & & & & 25 & & & & \\
\hline Whites & & & 29.8 & & & & 31.8 & & & & 12.0 & & 0.052 \\
\hline $\mathrm{AA}$ & & & 70.2 & & & & 68.2 & & & & 88.0 & & \\
\hline Poverty status & 245 & & & & 220 & & & & 25 & & & & 0.069 \\
\hline $\mathrm{PIR} \geq 125 \%$ & & & 37.1 & & & & 39.1 & & & & 20.0 & & \\
\hline PIR $<125 \%$ & & & 62.9 & & & & 60.9 & & & & 80.0 & & \\
\hline Hcy, $\log _{e}$ & 245 & & +2.26 & 0.02 & 220 & +2.19 & & 0.02 & 25 & +2.90 & & 0.05 & $<0.001$ \\
\hline
\end{tabular}

Abbreviations: HANDLS = Healthy Aging in Neighborhoods of Diversity Across the Life Span: Hcy = Homocysteine; NH = non-Hispanic; NHANES = National Health and Nutrition Examination Surveys; PIR = Poverty Income Ratio; ROC = Receiver Operating Characteristics analysis. ${ }^{1}$ All analyses, except for HANDLS, were adjusted for sampling design complexity, to obtain corrected standard errors for means and proportions. Means and proportions of study variables were compared across categories of Hcy $(0=$ normal, $1=$ hyperhomocysteinemic), using simple linear regression for continuous variables and logistic regression in which Hcy category was the outcome for categorical variables. $p$ value presented is associated with the regression coefficient. In NHANES, the regression models were also adjusted for sampling design complexity. Biochemical and hematological markers were compared by Hcy categories on their $\log _{\mathrm{e}}$-transformed scale (Mean, SE). However, the exponentiated mean is also presented for better clinical interpretation. 
Cross-validation (cv), adaptive and minBIC LASSO results are presented in Supplementary Table S1, using a random half sample of NHANES III, phase 2 and allowing for replication by sorting the sample by ID and setting a random seed to fixed value. Our findings indicated that $\log _{\mathrm{e}}$-transformed Hcy was associated with a number of biochemical and hematological indices, forcing adjustment for socio-demographic factors, most of which were shown to associate with elevated Hcy in Table 1. The result of the adaptive LASSO (initial model of choice) followed by backward elimination process is shown in Supplementary Table S2 for both continuous and binary Hcy outcomes, while accounting for survey design complexity. In the reduced logistic regression model, the finally selected predictors included: serum folate $(-)$, creatinine $(+)$, age $(+)$, serum vitamin B-12 $(-)$, aspartate aminotransferase $(+)$, alanine aminotransferase $(-)$, SUA $(+)$, mean cell hemoglobin, $\mathrm{MCH}(+)$, serum albumin $(+)$, serum vitamin $C(+)$, RDW $(+)$, alkaline phosphatase $(+)$, retinyl esters $(-)$ and serum cotinine (+). Additional control for five markers identified by LASSO logistic (adaptive method, Supplemental Table S1) and not by the LASSO linear (adaptive method) did not alter this finding. Of those, only 10 markers survived the selection criteria of AUC $>0.55$, and one (retinyl esters) was excluded due to missingness in recent waves. The remaining nine components were retained, with related ROC curves; estimated optimal cut-points for highest sensitivity/specificity are presented in Supplementary Figure S2. For NHANES 1999-2006, the ROC curves were similar for each of the nine components and cut-offs were comparable using the same criterion for elevated Hcy. The pooled NHANES similarly yielded a mid-range value of cut-points as indicated in Supplementary Figure S2.

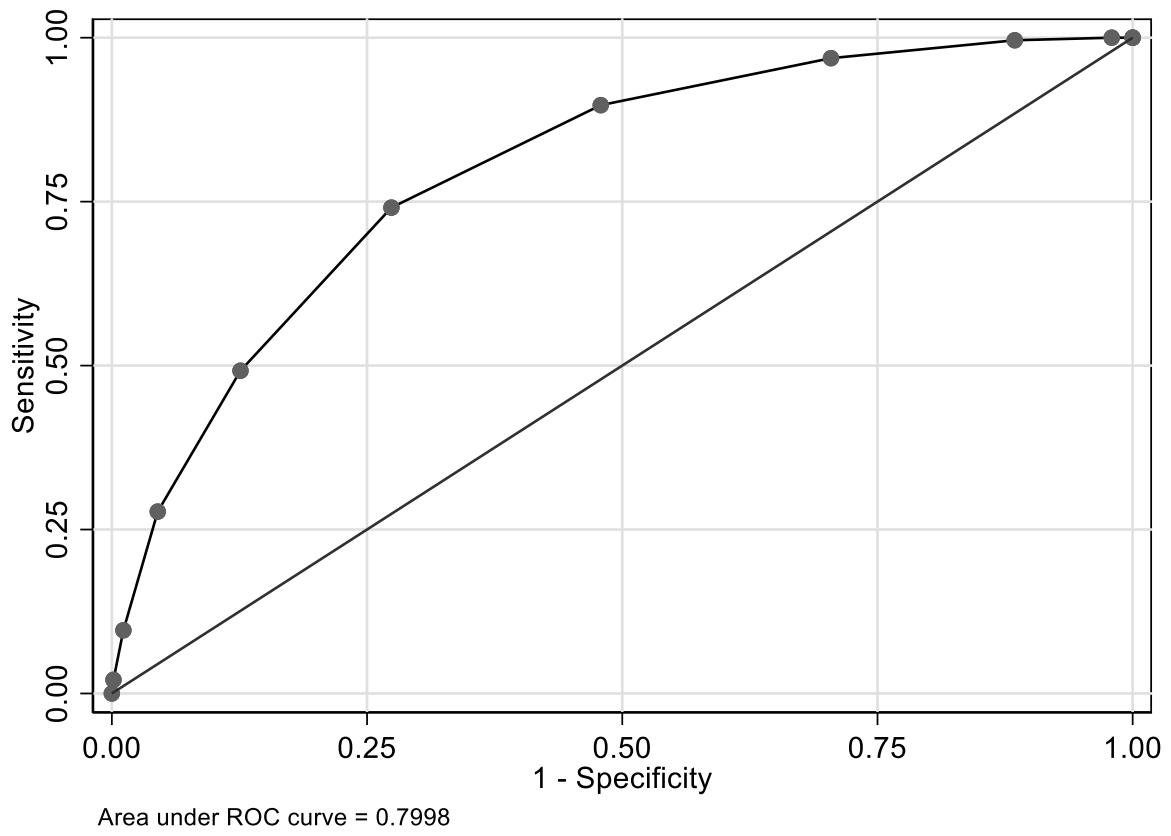

Figure 2. Nine-marker index (Index I) and its predictive value of elevated Hcy: ROC curves for pooled NHANES ${ }^{1}$. $^{1} n=13,822$; optimal cut-point was at 5; AUC $=0.799,95 \%$ CI: 0.785,0.814. Index I included binary biomarkers of elevated Hcy selected using LASSO and backward elimination. The full list of the nine components of Index I are: Age, serum folate, serum vitamin B-12, serum creatinine, red cell distribution width, mean cell hemoglobin, serum cotinine, serum uric acid and alkaline phosphatase. Cut-points for individual components are: serum folate, $\log _{\mathrm{e}}$, in $\mathrm{nmol} / \mathrm{L},<2.83$; serum creatinine, $\mathrm{Log}_{\mathrm{e}}$, in $\mu \mathrm{mol} / \mathrm{L} \geq 4.481$; older age, in years, $\geq 49$; serum vitamin $\mathrm{B}-12, \mathrm{Log}_{\mathrm{e}}$, in $\mathrm{pmol} / \mathrm{L},<5.74$; mean cell hemoglobin, $\log _{\mathrm{e}}$, in $p g, \geq 3.422$; red cell distribution width, $\log _{\mathrm{e}}$, in $\%, \geq 2.553$; serum uric acid, $\log _{\mathrm{e}}$, in $\mu \mathrm{mol} / \mathrm{L}, \geq 5.826$; serum alkaline phosphatase, $\log _{\mathrm{e}}$, in $\mu \mathrm{mol} / \mathrm{L}, \geq 4.356 \mathrm{U} / \mathrm{L}$; serum cotinine, $\log _{\mathrm{e}}$, in $\mathrm{ng} / \mathrm{mL},-0.579$.

The nine-component Index I predicted elevated Hcy with an AUC of 0.798 (95\% CI: 0.783,0.812) in the pooled NHANES data (Figure 2). Similarly, Index II, which excluded serum cotinine, thus 
including eight components, exhibited an AUC of 0.794; 95\% CI: 0.780,0.809 (Figure 3), indicating that when two values of Indices I and II were chosen at random, the lower value corresponded to a $\leq 14$ value of Hcy and the higher value to $>14$ value of Hcy, $\sim 80 \%$ of the time, suggesting a high predictive value of both indices for elevated Hcy. Optimal cut-point for both indices was 5 . For each binary index ( $\geq 5$ vs. $<5$ ) and in the pooled NHANES, (Table 2), the adjusted odds of elevated Hcy were increased 6.9-7.4-fold. Each of the nine binary components of Index I, when included into the model, simultaneously predicted elevated Hcy, independently increasing the odds by $>24 \%$ (higher RDW) up to 3.5-fold (lower serum folate).

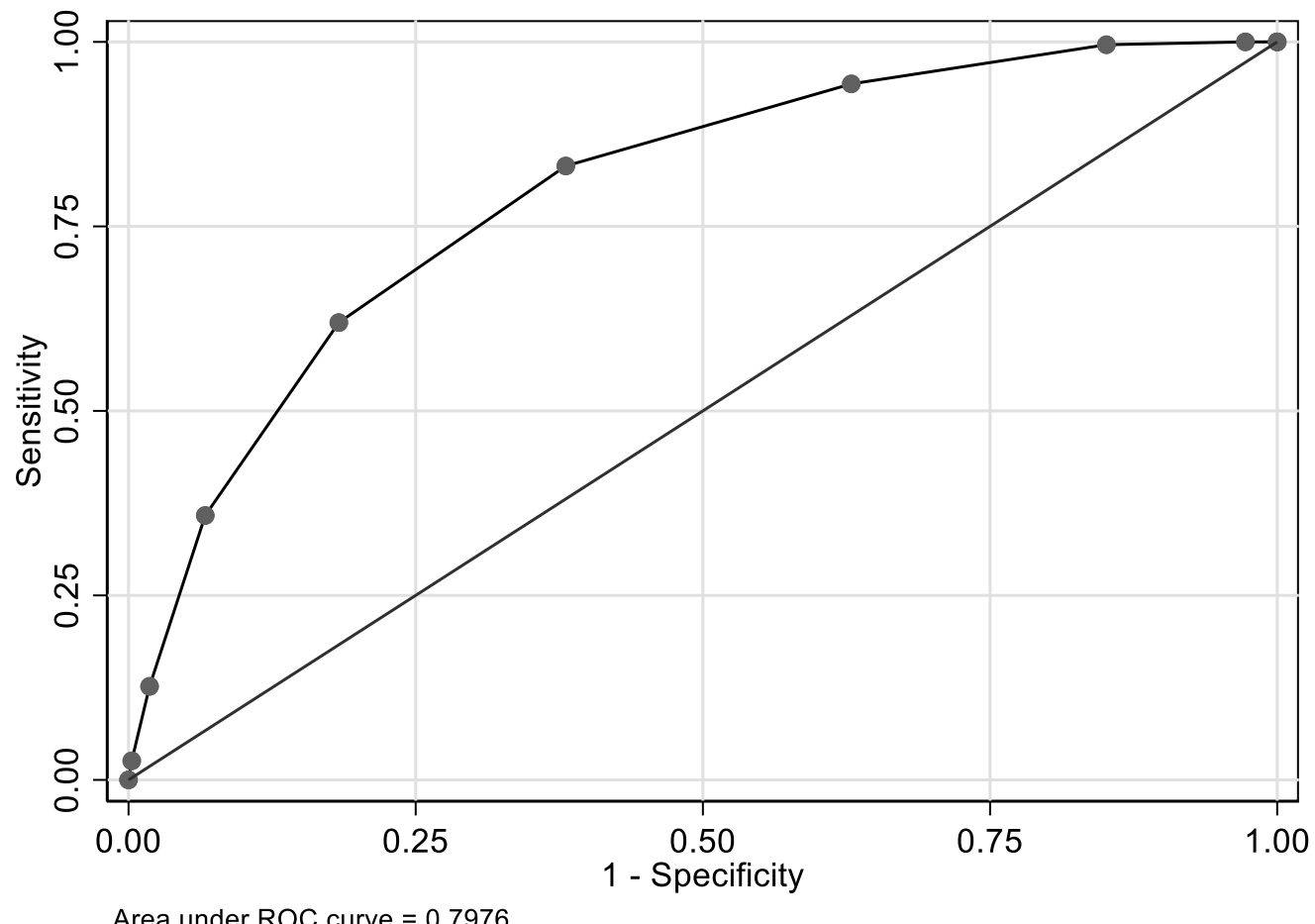

Figure 3. Eight-marker index (Index II) and its predictive value of elevated Hcy: pooled NHANES ${ }^{1}$. ${ }^{1} n=13,920$; optimal cut-point at 5; AUC $=0.798 ; 95 \%$ CI: $0.783,0.812$. The index consisted of a summation of all binary Index I biomarkers (See Figure 2 footnotes for cut-points), excluding blood cotinine, which was not available in HANDLS.

In the HANDLS sub-cohort analyses (Table 3), we found that Visit 1 Hcy was cross-sectionally associated, after multivariable adjustment, with lower serum folate, higher serum creatinine, lower serum vitamin B-12, and increases levels of SUA and alkaline phosphatase. Longitudinally, the multivariable adjusted mixed-effects regression model indicated that Visit $1 \mathrm{Hcy}$ was linked to faster rate of increase in serum creatinine and serum vitamin B -12, with a trend towards a direct association $(p<0.10)$ with rates of increase in RDW and alkaline phosphatase. Finally, Visit tHcy ( $\log _{\mathrm{e}}$-transformed) was found to be moderately and positively correlated with Visit 3 Index II, computed using pooled NHANES cut-points, with a Pearson's correlation $r=+0.34(n=81)$. The smoothed positive association is depicted in Figure 4, indicating a linear relationship for the range of the data. 
Table 2. Selected independent binary correlates and indices (I and II) of elevated homocysteine: Reduced multiple logistic regression models and model-specific area under the ROC curve; pooled NHANES III, phase 2 and 1999-2006 ${ }^{1}$.

\begin{tabular}{|c|c|c|c|}
\hline & \multicolumn{3}{|c|}{ Elevated Homocysteine } \\
\hline & OR & $95 \% \mathrm{CI}$ & $p$-Value \\
\hline \multicolumn{4}{|l|}{ Model 1: Binary predictors, $(n=14,739)$} \\
\hline Lower serum folate 2 & 3.49 & $(2.63,4.63)$ & $<0.001$ \\
\hline Higher serum creatinine $^{3}$ & 1.86 & $(1.51,2.29)$ & $<0.001$ \\
\hline Older age ${ }^{4}$ & 1.95 & $(1.56,2.44)$ & $<0.001$ \\
\hline Lower serum vitamin B-12 ${ }^{5}$ & 2.52 & $(1.98,3.21)$ & $<0.001$ \\
\hline Higher $\mathrm{MCH}^{6}$ & 1.60 & $(1.28,2.02)$ & $<0.001$ \\
\hline Higher RDW 7 & 1.24 & $(1.01,1.54)$ & 0.044 \\
\hline Higher SUA 8 & 1.67 & $(1.35,2.06)$ & $<0.001$ \\
\hline Higher alkaline phosphatase ${ }^{9}$ & 1.71 & $(1.35,2.15)$ & $<0.001$ \\
\hline Higher serum cotinine 10 & 1.77 & $(1.44,2.17)$ & $<0.001$ \\
\hline Model 2: Index I $\geq 5(n=14,739)$ & 7.43 & $(5.75,9.61)$ & $<0.001$ \\
\hline Model 3: Index II $\geq 5,(n=14,829)$ & 6.90 & $(5.37,8.84)$ & $<0.001$ \\
\hline
\end{tabular}

Abbreviations: HANDLS = Healthy Aging in Neighborhoods of Diversity Across the Life Span: Hcy = Homocysteine; NHANES = National Health and Nutrition Examination Surveys; ROC $=$ Receiver Operating Characteristics analysis. ${ }^{1}$ All elements of Indices I and II were Log transformed. Cut-points are determined using ROC analysis, using highest sensitivity/specificity combinations. Binary components are entered simultaneously in Model 1 . Model 2 includes Index I which sums binary variables: "lower serum folate" (1 = yes, 0 = no), "Higher serum creatinine", "Older age", "Lower serum vitamin B-12", "Higher MCH", "Higher RDW", "Higher SUA", "Higher alkaline phosphatase" and "Higher serum cotinine", with a possible range of 0-9. Model 3 included Index II which sums binary variables of Index I excluding "Higher blood lead" and "Higher serum cotinine". Possible range: 0-7. Cut-points for Indices I and II were also determined using ROC curve analysis, with an optimal cut-point selected using the highest sensitivity/specificity combination. ${ }^{2}$ Serum folate, Log ${ }_{\mathrm{e}}$ in $1 / \mathrm{L}<283,{ }^{3} \mathrm{Se}$ distribution width, $\log _{e}$, in $\%, \geq 2.553 ;^{8}$ Serum Uric Acid, Loge, in $\mu \mathrm{mol} / \mathrm{L}, \geq 5.826 ;{ }^{9}$ Serum alkaline phosphatase, Loge, in $\mu \mathrm{mol} / \mathrm{L}, \geq 4.356 \mathrm{U} / \mathrm{L} ;{ }^{10} \mathrm{Serum} \operatorname{cotinine}, \mathrm{Log}_{\mathrm{e}}$, in $\mathrm{ng} / \mathrm{mL},-0.579$. 
Table 3. Baseline serum homocysteine as a predictor of selected biochemical and hematological parameters at baseline and their change over time: mixed-effects linear regression models; HANDLS 2004-2018 ${ }^{1}$.

\begin{tabular}{|c|c|c|c|c|c|c|c|c|c|}
\hline \multirow[t]{2}{*}{ Outcome } & \multicolumn{2}{|c|}{ Intercept } & \multicolumn{2}{|l|}{ Time } & \multicolumn{2}{|c|}{ Hcy } & \multicolumn{2}{|c|}{ Hcy $\times$ Time } & \multirow[t]{2}{*}{ (n) $\mathrm{k}$} \\
\hline & $\gamma_{00} \pm \mathrm{SE}$ & $p$ & $\gamma_{10} \pm \mathrm{SE}$ & $p$ & $\gamma_{0 \mathrm{a}} \pm \mathrm{SE}$ & $p$ & $\gamma_{1 \mathrm{a}} \pm \mathrm{SE}$ & $p$ & \\
\hline Serum folate, $\mathrm{nmol} / \mathrm{L}$ & & & & & & & & & \\
\hline Model 1: Age-adjusted & $+33.6 \pm 1.2$ & $<0.001$ & $+0.11 \pm 0.16$ & 0.47 & $-0.21 \pm 0.32$ & 0.51 & $-0.05 \pm 0.05$ & 0.36 & $(243) \mathrm{k}=2.4$ \\
\hline Model 2: Socio-demographic adjusted & $+29.8 \pm 6.0$ & $<0.001$ & $+2.07 \pm 0.83$ & 0.012 & $-0.25 \pm 0.34$ & 0.45 & $-0.05 \pm 0.05$ & 0.37 & $(243) \mathrm{k}=2.4$ \\
\hline $\begin{array}{l}\text { Model 3: Multivariable-adjusted } \\
\text { Serum creatinine, } \mu \mathrm{mol} / \mathrm{L}\end{array}$ & $+35.1 \pm 6.2$ & $<0.001$ & $+1.39 \pm 0.87$ & 0.11 & $-1.41 \pm 0.43$ & 0.001 & $+0.03 \pm 0.07$ & 0.69 & $(227) \mathrm{k}=2.4$ \\
\hline Model 1: Age-adjusted & $+107.9 \pm 7.1$ & $<0.001$ & $-1.18 \pm 0.30$ & $<0.001$ & $+18.3 \pm 1.89$ & $<0.001$ & $+0.11 \pm 0.10$ & 0.24 & $(243) \mathrm{k}=2.4$ \\
\hline Model 2: Socio-demographic adjusted & $+100.1 \pm 37.1$ & 0.007 & $-3.40 \pm 1.50$ & 0.024 & $+18.3 \pm 2.00$ & $<0.001$ & $+0.14 \pm 0.10$ & 0.16 & $(243) \mathrm{k}=2.4$ \\
\hline $\begin{array}{l}\text { Model 3: Multivariable-adjusted } \\
\text { Serum vitamin B-12, } \mu \mathrm{mol} / \mathrm{L}\end{array}$ & $+117.7 \pm 35.1$ & 0.001 & $-3.93 \pm 1.60$ & 0.014 & $+19.8 \pm 2.01$ & $<0.001$ & $+0.24 \pm 0.12$ & 0.045 & $(227) \mathrm{k}=2.4$ \\
\hline Model 1: Age-adjusted & $+472 \pm 13$ & $<0.001$ & $-11.2 \pm 1.6$ & $<0.001$ & $-4.64 \pm 3.69$ & 0.20 & $+0.85 \pm 0.53$ & 0.11 & $(243) \mathrm{k}=2.4$ \\
\hline Model 2: Socio-demographic adjusted & $+334 \pm 68$ & $<0.001$ & $+6.1 \pm 8.6$ & 0.48 & $-5.19 \pm 3.80$ & 0.17 & $+0.87 \pm 0.55$ & 0.11 & $(243) \mathrm{k}=2.4$ \\
\hline $\begin{array}{l}\text { Model 3: Multivariable-adjusted } \\
\text { Mean cell hemoglobin, pg }\end{array}$ & $+307 \pm 69$ & $<0.001$ & $-8.0 \pm 8.9$ & 0.37 & $-11.0 \pm 4.9$ & 0.023 & $+1.66 \pm 0.69$ & 0.015 & $(227) \mathrm{k}=2.4$ \\
\hline Model 1: Age-adjusted & $+29.6 \pm 0.2$ & $<0.001$ & $-0.003 \pm 0.002$ & 0.56 & $+0.03 \pm 0.05$ & 0.53 & $-0.003 \pm 0.005$ & 0.56 & $(244) \mathrm{k}=2.4$ \\
\hline Model 2: Socio-demographic adjusted & $+30.9 \pm 0.9$ & $<0.001$ & $-0.04 \pm 0.10$ & 0.67 & $+0.02 \pm 0.05$ & 0.72 & $-0.004 \pm 0.005$ & 0.49 & $(244) \mathrm{k}=2.4$ \\
\hline $\begin{array}{l}\text { Model 3: Multivariable-adjusted } \\
\text { Red cell distribution width, \% }\end{array}$ & $+29.2 \pm 0.97$ & $<0.001$ & $+0.03 \pm 0.10$ & 0.78 & $+0.04 \pm 0.06$ & 0.50 & $+0.01 \pm 0.01$ & 0.42 & $(227) \mathrm{k}=2.4$ \\
\hline Model 1: Age-adjusted & $+13.8 \pm 0.11$ & $<0.001$ & $+0.12 \pm 0.01$ & $<0.001$ & $+0.05 \pm 0.03$ & 0.079 & $+0.007 \pm 0.003$ & 0.035 & $(244) \mathrm{k}=2.4$ \\
\hline Model 2: Socio-demographic adjusted & $+12.7 \pm 0.57$ & $<0.001$ & $+0.18 \pm 0.06$ & 0.002 & $+0.04 \pm 0.03$ & 0.16 & $+0.008 \pm 0.004$ & 0.021 & $(244) \mathrm{k}=2.4$ \\
\hline $\begin{array}{l}\text { Model 3: Multivariable-adjusted } \\
\text { Serum uric acid, } \mu \mathrm{mol} / \mathrm{L}\end{array}$ & $+12.7 \pm 0.5$ & $<0.001$ & $+0.19 \pm 0.06$ & 0.002 & $-0.04 \pm 0.04$ & 0.20 & $+0.009 \pm 0.005$ & 0.056 & $(227) \mathrm{k}=2.4$ \\
\hline Model 1: Age-adjusted & $+314.8 \pm 5.3$ & $<0.001$ & $+3.79 \pm 0.7$ & $<0.001$ & $+7.48 \pm 1.45$ & $<0.001$ & $-0.20 \pm 0.22$ & 0.36 & $(243) \mathrm{k}=2.4$ \\
\hline Model 2: Socio-demographic adjusted & $+226.8 \pm 26.7$ & $<0.001$ & $+2.80 \pm 3.51$ & 0.42 & $+5.30 \pm 1.48$ & $<0.001$ & $-0.12 \pm 0.23$ & 0.62 & $(243) \mathrm{k}=2.4$ \\
\hline $\begin{array}{l}\text { Model 3: Multivariable-adjusted } \\
\text { Serum alkaline phosphatase, U/L }\end{array}$ & $+228.6 \pm 27.0$ & $<0.001$ & $+2.01 \pm 3.61$ & 0.58 & $+8.11 \pm 1.88$ & $<0.001$ & $-0.01 \pm 0.29$ & 0.97 & $(227) \mathrm{k}=2.4$ \\
\hline Model 1: Age-adjusted & $+90.8 \pm 2.1$ & $<0.001$ & $-0.88 \pm 0.22$ & $<0.001$ & $+2.66 \pm 0.56$ & $<0.001$ & $-0.03 \pm 0.07$ & 0.70 & $(243) \mathrm{k}=2.4$ \\
\hline Model 2: Socio-demographic adjusted & $+70.7 \pm 10.5$ & $<0.001$ & $-0.39 \pm 1.19$ & 0.74 & $+2.54 \pm 0.58$ & $<0.001$ & $-0.002 \pm 0.08$ & 0.98 & $(243) \mathrm{k}=2.4$ \\
\hline Model 3: Multivariable-adjusted & $+75.2 \pm 10.7$ & $<0.001$ & $-0.27 \pm 1.23$ & 0.83 & $+2.72 \pm 0.74$ & $<0.001$ & $+0.16 \pm 0.10$ & 0.095 & $(227) \mathrm{k}=2.4$ \\
\hline
\end{tabular}

Abbreviations: HANDLS = Healthy Aging in Neighborhoods of Diversity Across the Life Span; Hcy = Homocysteine; $\mathrm{k}=$ mean number of observations/participant: $n=$ Number of participants. ${ }^{1}$ All selected biochemical and hematological markers were measured in SI units. Measures were not Log $\operatorname{eg}_{\mathrm{e}}$ transformed. Model 1 was adjusted for age, centered at 48.8 years. Model 2 was additionally adjusted for sex, race (African Americans vs. Whites), and poverty status (above vs. below poverty). Model 3 was additionally adjusted for all remaining biochemical and hematological measures that were selected. In Model 3, Folate was centered at 32.4, creatinine at 105.8, vitamin B-12 at 494.76, Mean cell hemoglobin at 29.46, red cell distribution width at 13.64 , serum uric acid at 306.2 , and alkaline phosphatase at 82 . 


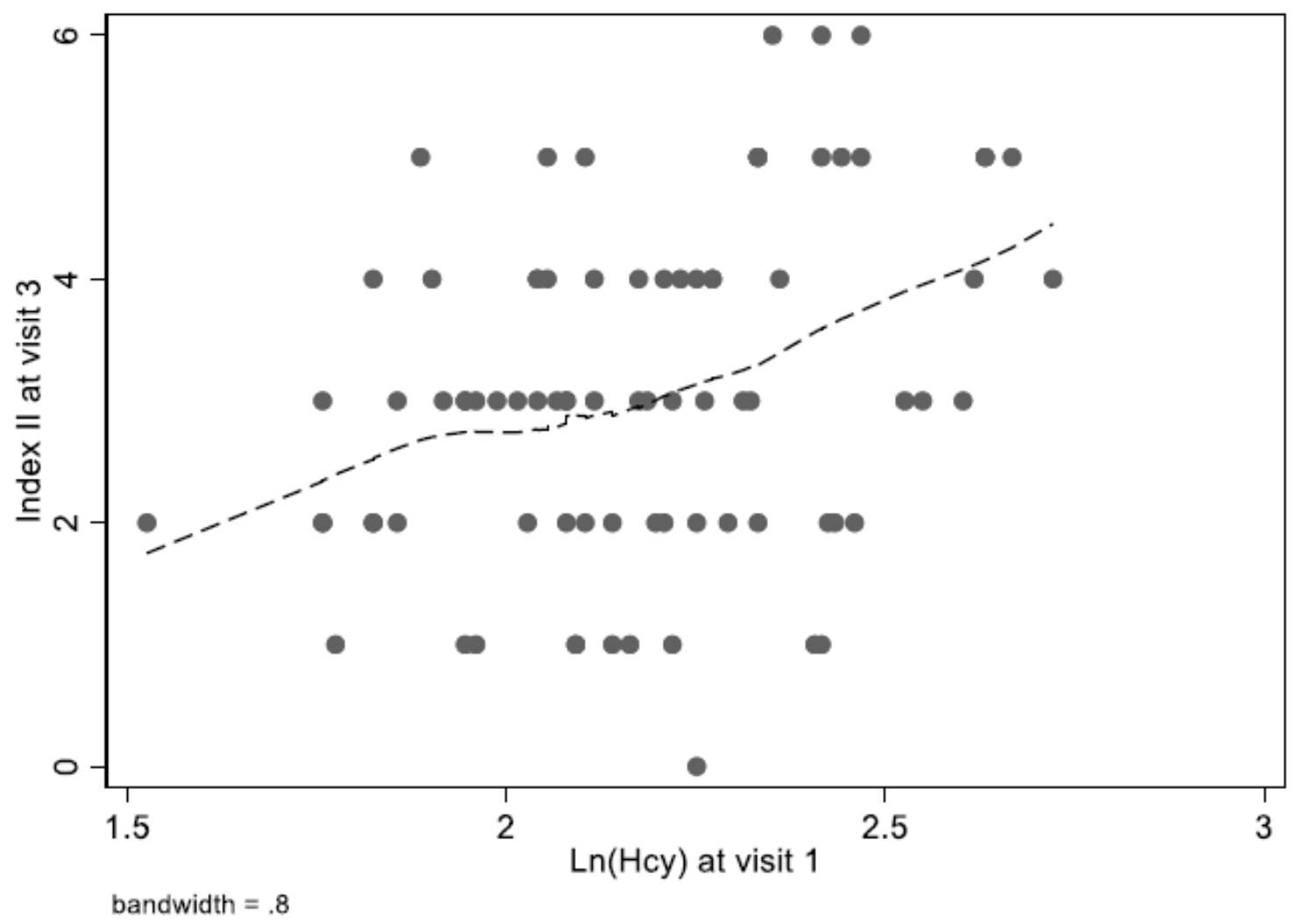

Figure 4. Lowess smoother of total Visit 1 homocysteine vs. Visit 3 Index II $(r=0.34, n=81)$ : HANDLS 2004-2009 (Hcy) and 2013-2018 (Index II) ${ }^{1}$. Abbreviations: HANDLS = Healthy Aging in Neighborhoods of Diversity Across the Life Span: Hcy = Homocysteine; NHANES = National Health and Nutrition Examination Surveys; ROC = Receiver Operating Characteristics analysis. ${ }^{1}$ Blood total $\mathrm{Hcy}$, in $\mu \mathrm{mol} / \mathrm{L}$, is $\mathrm{Log}_{\mathrm{e}}$-transformed and uncategorized. Index II was computed using cut-points obtained from the pooled NHANES ROC analysis. See Table 2 footnotes for details. Index II may range from 0 to 8 , and no cut-point was used in this analysis.

\section{Discussion}

Here we present data among middle-aged adults, validating a predictive index for Hcy $>14 \mu \mathrm{mol} / \mathrm{L}$ derived from independent biochemical and hematological correlates using modern techniques. The study uncovered up to nine independent predictors for elevated Hcy, some of which have been found to be correlated with each other (e.g., serum folate, $\mathrm{B}-12, \mathrm{MCH}$, and $\mathrm{RDW}$ ) in previous studies as well as with hyperhomocysteinemia. Both adjusted and unadjusted associations indicated that serum folate was the most predictive factor that was inversely related to elevated Hcy. Our findings of inverse associations of serum folate and cobalamin with elevated Hcy concentrations conform with earlier research using national data from pre-folate and post-folate fortification eras $[17,19,42]$. In fact, the two previous NHANES studies (III, phase 2:1991-1994 and 1999-2004) reported comparable findings, despite examining pre-selected factors, rather than exploring all available biochemical and hematological markers. The NHANES III study concluded that serum creatinine and cobalamin concentrations showed the strongest and weakest association with blood Hcy, respectively [19]. Notably, folate and vitamin B-12 were inversely related to Hcy. Men had higher mean Hcy than women, along with lower concentrations of serum folate, red blood cell (RBC) folate, and serum vitamin B12. The NHANES 1999-2004 study concluded that blood Hcy concentration was 9.7\% higher in men vs. woman [17], and was directly related to systolic blood pressure, serum creatinine, and serum cotinine, while being inversely correlated with serum folate levels, RBC folate, and serum vitamin B-12, and positively correlated with methylmalonic acid (MMA) concentration [17]. While those associations were largely replicated in our study, age and not sex was retained in the model upon 
backward elimination. Moreover, our study excluded other lifestyle or health-related factors, selecting only biochemical and hematological indices measured in NHANES III, phase 2 (1991-1994) along with Hcy. Consequently, MMA was excluded due to its unavailability in the NHANES III phase 2 data from among the 82 selected biomarkers.

Among retained correlates, SUA was strongly related to Hcy. In a retrospective cohort study $(n=16,477$, age: 20-80 years), elevated SUA was previously directly associated with hyperhomocysteinemia, whereby the fully adjusted association remained significant only among men $(\mathrm{OR}=1.5 ; 95 \% \mathrm{CI}, 1.3,1.7 ; p<0.001)$ [43]. These results are comparable to our findings, whereby SUA $\geq 339 \mu \mathrm{mol} / \mathrm{L}$ was associated with a 1.67-fold increase in the odds of hyperhomocysteinemia (>14 $\mu \mathrm{mol} / \mathrm{L}$ ), with a 95\% CI: 1.35,2.06.

Hyperuricemia can be modified with diet as well, including reduced alcohol, red meat and sugar consumption $[44,45]$.

High $\mathrm{MCH}$ is commonly a sign of macrocytic anemia (i.e., enlarged RBCs) subsequent to folate or vitamin B-12 deficiency [46], though it may also result from liver diseases [46]. Thus, Hcy may well be a correlate of higher $\mathrm{MCH}$, resulting from any or both vitamin deficiencies; and a higher Hcy may result in higher $\mathrm{MCH}$ over time. Nevertheless, our findings indicated that although an independent correlate of elevated $\mathrm{Hcy}, \mathrm{MCH}$ was not among the strongest predictors based on ROC analyses. Furthermore, our longitudinal analyses did not indicate that baseline Hcy was associated with faster increase in $\mathrm{MCH}$ over time. Nevertheless, larger studies are needed to corroborate these findings. Elevated RDW, reflecting RBC size variability (i.e., anisocytosis), independently predicted chronic disease morbidity and mortality [47-51]. Unlike MCH, RDW was previously studied in relation to hyperhomocysteinemia [22,52], and was found to be directly related to elevated Hcy in one cross-sectional study of middle-aged Chinese adults, independently of age, neutrophil count, mean corpuscular volume, and hemoglobin [22]. Another larger cross-sectional study of 5554 adults (18-64 years), however, failed to detect this independent RDW-Hcy [52]. Our findings indicated that elevated MCH was in fact more strongly associated with elevated Hcy (OR $=1.60,95 \%$ CI: 1.28,2.02) when compared with RDW (OR $=1.24,95 \%$ CI:1.01,1.54). Thus, elevated Hcy may be a stronger marker of enlarged RBCs than of anisocytosis. Nevertheless, our longitudinal analysis has shown that Hcy in its continuous form predicted RDW to a greater extent than $\mathrm{MCH}$, and was associated with a faster rate of increase in RDW over time.

Moreover, serum bone alkaline phosphatase, a marker of biliary inflammation and cholelithiasis [53], was shown to be up-regulated in vitamin B-6 deficiency [25,54]. As stated earlier, Hcy was previously inversely corelated with vitamin B-6 status [9-14], as the latter is directly involved in OCM. Our study is to date the first to show that higher blood Hcy is associated with elevated alkaline phosphatase, both cross-sectionally and longitudinally. Thus, although the main modifiers of Hcy are B-vitamins, particularly folate and vitamin B-12, liver enzymes are correlates of Hcy that can be modulated with reduced alcohol consumption [55], and alkaline phosphatase in particular is a key mediator in the reported association between Hcy and reduction in bone mineral density associated with osteoporosis among postmenopausal women [56,57]. Thus, Hcy may be merely a marker of certain health outcomes, while liver enzyme elevations can act as the main causal pathway.

Several studies have indicated that smokers had more elevated blood Hcy than non-smokers, independently of other factors, while having lower serum levels of folate and vitamin B-12 [23]. Among self-reported never smokers $>20$ years of age [NHANES III, $n=3232$ ], serum cotinine quartiles were independently and linearly associated with blood Hcy, as were age, being male, being non-white, and having lower sum folate or serum B-12 [58]. Serum cotinine was also among key predictors of elevated Hcy in studies examining multiple correlates in earlier and more recent NHANES [17,19]. Thus, stopping cigarette smoking may have an effect of reducing the risk of elevated Hcy. Nevertheless, controlled randomized trials are needed to ascertain a causal association. It is worth noting that both serum cotinine and urinary cadmium were linked to recent smoking [59,60].

Several notable study strengths include the novel coupled use of machine learning and ROC analyses to select independent predictors for elevated Hcy and subsequently create combined indices 
and conduct multivariable regression models. The initial analysis screened over 82 biochemical and hematological biomarkers, and our confidence in the predictive indices was enhanced by validation between cross-sectional national data and an independent longitudinal study of urban adults. The LASSO linear model was used to obtain a first set of predictors for continuous $\log _{\mathrm{e}}$-transformed Hcy, which were then applied to the binary outcome, given that the $14 \mu \mathrm{mol} / \mathrm{L}$ cutoff to define elevated Hcy might be considered arbitrary for some health outcomes, aside from AD. Thus, our goal was to limit the set of markers to those that independently predicted Hcy, both in its continuous and categorical form.

Among the limitations, the threshold used for Hcy of $14 \mu \mathrm{mol} / \mathrm{L}$ in our study, while being used by others previously, may be sub-optimal in some samples, given their different levels of mean Hcy, particularly given the decreasing prevalence rates between pre- and post-folate fortification. Thus, even though cutoffs for predictors were comparable at optimal sensitivity and specificity between NHANES waves, the predictive values may have differed between those two waves, with expected higher positive predictive value at higher prevalence of elevated Hcy (i.e., NHANES III, phase 2) and vice versa for the negative predictive value. Second, Hcy measurement, while comparable between waves, used different techniques between NHANES III, phase 2 and the more recent NHANES, potentially affecting the validity of the cut-point used between those two waves. Nevertheless, given that comparable biomarker optimal cut-points were obtained between NHANES waves through ROC analyses for Hcy $>14 \mu \mathrm{mol} / \mathrm{L}$, measurement errors ascribed to differential use of techniques (HPLC vs. immunoassay) was assumed minimal. Attempts to calibrate those two methods are needed in future studies with repeat measures using both methods. Finally, our key findings and the indices derived from ROC analyses may be applicable only at mid-life, a time window whereby cardiovascular and neurodegenerative diseases can be prevented through Hcy-reducing interventions. Nevertheless, future studies should examine those relationships and validate those indices among older adults aged $\geq 65$ years.

In sum, we provide evidence of internal and external validity of indices composed of several biochemical and hematological markers that are strongly associated with elevated Hcy, which may be used as proxies in future longitudinal studies. Components of those indices that are amenable to intervention (e.g., folate and B-12 supplementation, alcohol consumption which affects both liver enzymes and uric acid, cigarette smoking) should also be studied as alternative pathways for which elevated Hcy can affect cardiovascular and neurodegenerative disease trajectories.

Supplementary Materials: The following are available online at http://www.mdpi.com/2072-6643/12/4/950/ s1, Supplementary Figure S1. Participant flowchart. Supplementary Figure S2. ROC curves of selected correlates of elevated homocysteine ( $>14 \mu \mathrm{mol} / \mathrm{L})$ and cut-point determination: NHANES III, phase 2; NHANES 1999-2006 and pooled NHANES. Supplemental Methods 1: Biochemical and Hematological Indices: NHANES III, phase 2: 1991-1994. Supplemental Methods 2: Biochemical and Hematological Indices Selected for NHANES 1999-2006. Supplemental Methods 3: Biochemical and Hematological Indices Selected for HANDLS 2004-2018. Supplemental Methods 4: Description of Time-Interval Mixed-effects linear regression models, HANDLS 2004-2018. Supplementary Table S1. Predictive models of serum homocysteine (Loge transformed): CV, minimum BIC and adaptive linear LASSO for NHANES III, phase 2: training half-sample results and consistency with testing half-sample, 30-65 y. Supplementary Table S2. Reduced selected LASSO model for biochemical and hematological predictors (Loge transformed and z-scored) of Hcy (Loge transformed and z-scored) and elevated Hcy (equivalent to Hcy $>14 \mu \mathrm{mol} / \mathrm{L}$ ), adjusting for socio-demographic factors: NHANES III, phase 2; ages 30-65 y.

Author Contributions: M.A.B. contributed to the study concept, planned analysis, conducted data management and statistical analysis, conducted literature review, wrote and revised the manuscript. H.A.B. planned analysis, assisted in data management and statistical analysis, conducted literature review, wrote and revised parts of the manuscript. P.H.M. planned analysis, conducted literature search and review, wrote and revised parts of the manuscript. S.H. planned analysis, conducted literature search and review, wrote parts of the manuscript, revised the manuscript. J.A.C. conducted literature review, wrote and revised parts of the manuscript. M.K.E. acquired data, wrote and revised parts of the manuscript. A.B.Z. acquired data, planned analysis, wrote and revised parts of the manuscript. All authors have read and agreed to the published version of the manuscript.

Funding: This work was supported in part by the Intramural Research Program of the NIH, National institute on Aging. 
Acknowledgments: We would like to thank Megan Williams and Nicolle Mode (NIA/NIH/IRP) for internally reviewing our manuscript. The authors declare no conflict of interest.

Conflicts of Interest: The authors declare no conflict of interest.

\section{Abbreviations}

$\begin{array}{ll}\text { AUC } & \text { Area under the curve } \\ \text { BIC } & \text { Bayesian Information Criterion } \\ \text { CDC } & \text { Centers for Disease Control and Prevention } \\ \text { cv } & \text { Cross-validation } \\ \text { HANDLS } & \text { Healthy Aging in Neighborhood of Diversity Across the Life Span } \\ \text { Hcy } & \text { Homocysteine } \\ \text { LASSO } & \text { least absolute shrinkage and selection operator } \\ \text { LOWESS } & \text { Locally weighted regression } \\ \text { MCH } & \text { Mean cell hemoglobin } \\ \text { MEC } & \text { mobile examination center } \\ \text { MMA } & \text { methylmalonic acid } \\ \text { MRV } & \text { Medical Research Vehicles } \\ \text { MTHF } & \text { N-5-methyl-tetrahydrofolate } \\ \text { NCHS } & \text { National Center for Health Statistics } \\ \text { NHANES } & \text { National Health and Nutrition Examination Surveys } \\ \text { RDW } & \text { Red cell distribution width } \\ \text { ROC } & \text { Receiver Operating Characteristic } \\ \text { SAH } & \text { S-adenosylhomocysteine } \\ \text { SAM } & \text { S-adenosylemethionine } \\ \text { SUA } & \text { Serum Uric Acid }\end{array}$

\section{References}

1. Selhub, J. Public health significance of elevated homocysteine. Food Nutr. Bull. 2008, 29, S116-S125. [CrossRef] [PubMed]

2. Seshadri, S.; Beiser, A.; Selhub, J.; Jacques, P.F.; Rosenberg, I.H.; D'Agostino, R.B.; Wilson, P.W.; Wolf, P.A. Plasma homocysteine as a risk factor for dementia and Alzheimer's disease. N. Engl. J. Med. 2002, 346, 476-483. [CrossRef] [PubMed]

3. Clarke, R.; Smith, A.D.; Jobst, K.A.; Refsum, H.; Sutton, L.; Ueland, P.M. Folate, vitamin B12, and serum total homocysteine levels in confirmed Alzheimer disease. Arch. Neurol. 1998, 55, 1449-1455. [CrossRef]

4. Beydoun, M.A.; Beydoun, H.A.; Gamaldo, A.A.; Teel, A.; Zonderman, A.B.; Wang, Y. Epidemiologic studies of modifiable factors associated with cognition and dementia: Systematic review and meta-analysis. BMC Public Health 2014, 14, 643. [CrossRef] [PubMed]

5. Selhub, J.; Jacques, P.F.; Rosenberg, I.H.; Rogers, G.; Bowman, B.A.; Gunter, E.W.; Wright, J.D.; Johnson, C.L. Serum total homocysteine concentrations in the third National Health and Nutrition Examination Survey (1991-1994): Population reference ranges and contribution of vitamin status to high serum concentrations. Ann. Intern. Med. 1999, 131, 331-339. [CrossRef] [PubMed]

6. Booth, G.L.; Wang, E.E. Preventive health care, 2000 update: Screening and management of hyperhomocysteinemia for the prevention of coronary artery disease events. The Canadian Task Force on Preventive Health Care. CMAJ 2000, 163, 21-29.

7. Marti-Carvajal, A.J.; Sola, I.; Lathyris, D.; Dayer, M. Homocysteine-lowering interventions for preventing cardiovascular events. Cochrane Database Syst. Rev. 2017, 8, CD006612. [CrossRef]

8. Selhub, J.; Miller, J.W. The pathogenesis of homocysteinemia: Interruption of the coordinate regulation by S-adenosylmethionine of the remethylation and transsulfuration of homocysteine. Am. J. Clin. Nutr. 1992, 55, 131-138. [CrossRef]

9. Hatzis, C.M.; Bertsias, G.K.; Linardakis, M.; Scott, J.M.; Kafatos, A.G. Dietary and other lifestyle correlates of serum folate concentrations in a healthy adult population in Crete, Greece: A cross-sectional study. Nutr. J. 2006, 5, 5. [CrossRef] 
10. Manavifar, L.; Nemati Karimooy, H.; Jamali, J.; Talebi Doluee, M.; Shirdel, A.; Nejat Shokohi, A.; Fatemi Nayyeri, M. Homocysteine, Cobalamin and Folate Status and their Relations to Neurocognitive and Psychological Markers in Elderly in Northeasten of Iran. Iran J. Basic Med. Sci. 2013, 16, 772-780.

11. Song, J.H.; Park, M.H.; Han, C.; Jo, S.A.; Ahn, K. Serum Homocysteine and Folate Levels are Associated With Late-life Dementia in a Korean Population. Osong Public Health Res. Perspect. 2010, 1, 17-22. [CrossRef] [PubMed]

12. Yang, X.; Gao, F.; Liu, Y. Association of homocysteine with immunological-inflammatory and metabolic laboratory markers and factors in relation to hyperhomocysteinaemia in rheumatoid arthritis. Clin. Exp. Rheumatol. 2015, 33, 900-903. [PubMed]

13. Cheng, C.H.; Huang, Y.C.; Chen, F.P.; Chou, M.C.; Tsai, T.P. B-vitamins, homocysteine and gene polymorphism in adults with fasting or post-methionine loading hyperhomocysteinemia. Eur. J. Nutr. 2008, 47, 491-498. [CrossRef]

14. Selhub, J.; Jacques, P.F.; Wilson, P.W.; Rush, D.; Rosenberg, I.H. Vitamin status and intake as primary determinants of homocysteinemia in an elderly population. JAMA 1993, 270, 2693-2698. [CrossRef] [PubMed]

15. Rozen, R. Genetic predisposition to hyperhomocysteinemia: Deficiency of methylenetetrahydrofolate reductase (MTHFR). Thromb. Haemost. 1997, 78, 523-526. [CrossRef] [PubMed]

16. Ohishi, T.; Fujita, T.; Suzuki, D.; Nishida, T.; Asukai, M.; Matsuyama, Y. Serum homocysteine levels are affected by renal function during a 3-year period of minodronate therapy in female osteoporotic patients. J. Bone Miner. Metab. 2019, 37, 319-326. [CrossRef] [PubMed]

17. Ganji, V.; Kafai, M.R. Demographic, lifestyle, and health characteristics and serum B vitamin status are determinants of plasma total homocysteine concentration in the post-folic acid fortification period, 1999-2004. J. Nutr. 2009, 139, 345-352. [CrossRef]

18. Francis, M.E.; Eggers, P.W.; Hostetter, T.H.; Briggs, J.P. Association between serum homocysteine and markers of impaired kidney function in adults in the United States. Kidney Int. 2004, 66, 303-312. [CrossRef]

19. Ganji, V.; Kafai, M.R.; Third National, H.; Nutrition Examination, S. Demographic, health, lifestyle, and blood vitamin determinants of serum total homocysteine concentrations in the third National Health and Nutrition Examination Survey, 1988-1994. Am. J. Clin. Nutr. 2003, 77, 826-833. [CrossRef]

20. Fukagawa, N.K.; Martin, J.M.; Wurthmann, A.; Prue, A.H.; Ebenstein, D.; O’Rourke, B. Sex-related differences in methionine metabolism and plasma homocysteine concentrations. Am. J. Clin. Nutr. 2000, 72, 22-29. [CrossRef]

21. Sadre-Marandi, F.; Dahdoul, T.; Reed, M.C.; Nijhout, H.F. Sex differences in hepatic one-carbon metabolism. BMC Syst. Biol. 2018, 12, 89. [CrossRef] [PubMed]

22. Peng, Y.F.; Pan, G.G. Red blood cell distribution width predicts homocysteine levels in adult population without vitamin B12 and folate deficiencies. Int. J. Cardiol. 2017, 227, 8-10. [CrossRef] [PubMed]

23. Haj Mouhamed, D.; Ezzaher, A.; Neffati, F.; Douki, W.; Najjar, M.F. Effect of cigarette smoking on plasma homocysteine concentrations. Clin. Chem. Lab. Med. 2011, 49, 479-483. [CrossRef]

24. Lippi, G.; Salvagno, G.L.; Targher, G.; Montagnana, M.; Guidi, G.C. Plasma gamma-glutamyl transferase activity predicts homocysteine concentration in a large cohort of unselected outpatients. Intern. Med. 2008, 47, 705-707. [CrossRef]

25. Tanaka, T.; Scheet, P.; Giusti, B.; Bandinelli, S.; Piras, M.G.; Usala, G.; Lai, S.; Mulas, A.; Corsi, A.M.; Vestrini, A.; et al. Genome-wide association study of vitamin B6, vitamin B12, folate, and homocysteine blood concentrations. Am. J. Hum. Genet. 2009, 84, 477-482. [CrossRef] [PubMed]

26. Center for Disease Control and Prevention (CDC). National Health and Nutrition Examination Survey. Available online: http://www.cdc.gov/nchs/nhanes.htm (accessed on 25 October 2019).

27. NCHS. Plan and Operation of the Third National Health and Nutrition Examination Survey, 1988-1994; NCHS: Highlandsville, MD, USA, 1994.

28. Crider, K.S.; Bailey, L.B.; Berry, R.J. Folic acid food fortification-its history, effect, concerns, and future directions. Nutrients 2011, 3, 370-384. [CrossRef]

29. Center for Disease Control and Prevention (CDC). Laboratory Procedures Used for the Third National Health and Nutrition Examination Survey (NHANES III), 1988-1994. Available online: https://www.cdc.gov/nchs/ data/nhanes/nhanes3/cdrom/nchs/manuals/labman.pdf (accessed on 25 October 2019). 
30. Evans, M.K.; Lepkowski, J.M.; Powe, N.R.; LaVeist, T.; Kuczmarski, M.F.; Zonderman, A.B. Healthy aging in neighborhoods of diversity across the life span (HANDLS): Overcoming barriers to implementing a longitudinal, epidemiologic, urban study of health, race, and socioeconomic status. Ethn. Dis. 2010, 20, 267-275.

31. Araki, A.; Sako, Y. Determination of free and total homocysteine in human plasma by high-performance liquid chromatography with fluorescence detection. J. Chromatogr. 1987, 422, 43-52. [CrossRef]

32. Abbott Homocysteine (HCY) assay package insert fo IMX Analyzer. Available online: https://www.cdc.gov/ nchs/data/nhanes/nhanes_01_02/106_b_met_homocysteine_IMX.pdf (accessed on 25 October 2019).

33. Pernet, P.; Lasnier, E.; Vaubourdolle, M. Evaluation of the AxSYM homocysteine assay and comparison with the IMx homocysteine assay. Clin. Chem. 2000, 46, 1440-1441. [CrossRef]

34. Laboratories, B.R. Instruction Manual, Bio-Rad Quantaphase Folate Radioassay Kit; Bio-Rad Laboratories: Hercules, CA, USA, 1987.

35. Centers for Disease Control and Prevention (CDC). National Health and Nutrition Examination Surveys (NHANES 2005-06): Description of Laboratory Methodology: Vitamin B-12. Available online: https: //wwwn.cdc.gov/Nchs/Nhanes/2005-2006/B12_D.htm (accessed on 15 February 2019).

36. Centers for Disease Control and Prevention (CDC). National Health and Nutrition Examination Surveys (NHANES 2005-06): Description of Laboratory Methodology: Folate. Available online: https://wwwn.cdc. gov/Nchs/Nhanes/2005-2006/FOLATE_D.htm (accessed on 15 February 2019).

37. Diagnostics, Q. Vitamin B-12 (Cobalamin) and Folate Panel. Available online: https://testdirectory. questdiagnostics.com/test/test-detail/7065/vitamin-b12-cobalamin-and-folate-panel-serum?cc=MASTER (accessed on 21 October 2019).

38. STATA. Statistics/Data Analysis: Release 16.0; Stata Corporation: College Station, TX, USA, 2019.

39. Zou, H. The adaptive Lasso and it oracle properties. J. Am. Stat. Assoc. 2006, 101, 1418-1428. [CrossRef]

40. Albeck, M.J.; Borgesen, S.E. ROC-curve analysis. A statistical method for the evaluation of diagnostic tests. Ugeskr Laeger 1990, 152, 1650-1653. [PubMed]

41. Soreide, K. Receiver-operating characteristic (ROC) curve analysis in diagnostic, prognostic and predictive biomarker research. J. Clin. Pathol. 2008. [CrossRef]

42. Jacques, P.F.; Bostom, A.G.; Wilson, P.W.; Rich, S.; Rosenberg, I.H.; Selhub, J. Determinants of plasma total homocysteine concentration in the Framingham Offspring cohort. Am. J. Clin. Nutr. 2001, 73, 613-621. [CrossRef] [PubMed]

43. Cohen, E.; Levi, A.; Vecht-Lifshitz, S.E.; Goldberg, E.; Garty, M.; Krause, I. Assessment of a possible link between hyperhomocysteinemia and hyperuricemia. J. Investig. Med. 2015, 63, 534-538. [CrossRef] [PubMed]

44. Beydoun, M.A.; Canas, J.A.; Fanelli-Kuczmarski, M.T.; Tajuddin, S.M.; Evans, M.K.; Zonderman, A.B. Genetic risk scores, sex and dietary factors interact to alter serum uric acid trajectory among African-American urban adults. Br. J. Nutr. 2017, 117, 686-697. [CrossRef]

45. Beydoun, M.A.; Fanelli-Kuczmarski, M.T.; Canas, J.A.; Beydoun, H.A.; Evans, M.K.; Zonderman, A.B. Dietary factors are associated with serum uric acid trajectory differentially by race among urban adults. Br. J. Nutr. 2018, 120, 935-945. [CrossRef]

46. Aslinia, F.; Mazza, J.J.; Yale, S.H. Megaloblastic anemia and other causes of macrocytosis. Clin. Med. Res. 2006, 4, 236-241. [CrossRef]

47. Li, N.; Zhou, H.; Tang, Q. Red Blood Cell Distribution Width: A Novel Predictive Indicator for Cardiovascular and Cerebrovascular Diseases. Dis. Markers 2017, 2017, 7089493. [CrossRef]

48. Tajuddin, S.M.; Nalls, M.A.; Zonderman, A.B.; Evans, M.K. Association of red cell distribution width with all-cause and cardiovascular-specific mortality in African American and white adults: A prospective cohort study. J. Transl. Med. 2017, 15, 208. [CrossRef]

49. Hoffmann, J.J. Red cell distribution width and mortality risk. Clin. Chim. Acta 2012, 413, 824-825. [CrossRef]

50. Perlstein, T.S.; Weuve, J.; Pfeffer, M.A.; Beckman, J.A. Red blood cell distribution width and mortality risk in a community-based prospective cohort. Arch. Intern. Med. 2009, 169, 588-594. [CrossRef] [PubMed]

51. Patel, K.V.; Semba, R.D.; Ferrucci, L.; Newman, A.B.; Fried, L.P.; Wallace, R.B.; Bandinelli, S.; Phillips, C.S.; Yu, B.; Connelly, S.; et al. Red cell distribution width and mortality in older adults: A meta-analysis. J. Gerontol. A Biol. Sci. Med. Sci. 2010, 65, 258-265. [CrossRef] [PubMed] 
52. Margalit, I.; Cohen, E.; Goldberg, E.; Krause, I. Reconsidering the relation between serum homocysteine and red blood cell distribution width: A cross-sectional study of a large cohort. Biomarkers 2018, 23, 483-486. [CrossRef] [PubMed]

53. Poupon, R. Liver alkaline phosphatase: A missing link between choleresis and biliary inflammation. Hepatology 2015, 61, 2080-2090. [CrossRef]

54. Loohuis, L.M.; Albersen, M.; de Jong, S.; Wu, T.; Luykx, J.J.; Jans, J.J.M.; Verhoeven-Duif, N.M.; Ophoff, R.A. The Alkaline Phosphatase (ALPL) Locus Is Associated with B6 Vitamer Levels in CSF and Plasma. Genes (Basel) 2018, 10, 8. [CrossRef]

55. Wannamethee, S.G.; Shaper, A.G. Cigarette smoking and serum liver enzymes: The role of alcohol and inflammation. Ann. Clin. Biochem. 2010, 47, 321-326. [CrossRef]

56. Bailey, R.L.; Looker, A.C.; Lu, Z.; Fan, R.; Eicher-Miller, H.A.; Fakhouri, T.H.; Gahche, J.J.; Weaver, C.M.; Mills, J.L. B-vitamin status and bone mineral density and risk of lumbar osteoporosis in older females in the United States. Am. J. Clin. Nutr. 2015, 102, 687-694. [CrossRef]

57. Nakamura, Y.; Suzuki, T.; Kato, H. Serum bone alkaline phosphatase is a useful marker to evaluate lumbar bone mineral density in Japanese postmenopausal osteoporotic women during denosumab treatment. Ther. Clin. Risk Manag. 2017, 13, 1343-1348. [CrossRef]

58. Kim, D.B.; Oh, Y.S.; Yoo, K.D.; Lee, J.M.; Park, C.S.; Ihm, S.H.; Jang, S.W.; Shim, B.J.; Kim, H.Y.; Seung, K.B.; et al. Passive smoking in never-smokers is associated with increased plasma homocysteine levels. Int. Heart J. 2010, 51, 183-187. [CrossRef]

59. Lee, W.; Lee, S.; Roh, J.; Won, J.U.; Yoon, J.H. The Association between Involuntary Smoking Exposure with Urine Cotinine Level and Blood Cadmium Level in General Non-Smoking Populations. J. Korean Med. Sci. 2017, 32, 568-575. [CrossRef]

60. Sanchez-Rodriguez, J.E.; Bartolome, M.; Canas, A.I.; Huetos, O.; Navarro, C.; Rodriguez, A.C.; Arribas, M.; Esteban, M.; Lopez, A.; Castano, A. Anti-smoking legislation and its effects on urinary cotinine and cadmium levels. Environ. Res. 2015, 136, 227-233. [CrossRef] [PubMed]

(C) 2020 by the authors. Licensee MDPI, Basel, Switzerland. This article is an open access article distributed under the terms and conditions of the Creative Commons Attribution (CC BY) license (http://creativecommons.org/licenses/by/4.0/). 